\title{
Rasch Model Application on Character Development Instrument for Elementary School Students
}

\author{
Lutfi Nur*, Luthfi Ainun Nurani and Dodi Suryana \\ Universitas Pendidikan Indonesia, Bandung, Indonesia \\ Aslina Ahmad \\ Universitas Pendidikan Sultan Idris, Malaysia
}

\begin{abstract}
This research was motivated by the current conditions that require the younger generation to have good character values or ethics. Even nowadays humans begin to indicate that some professions will be lost and replaced by machines. However, the value of good character and ethics will not be able to be replaced by the machine. The purpose of this research is to ascertain how far elementary school students have good character values or ethics so that we can spot a trend whether character values are degenerating, getting better or simply staying the same. The research respondents were the fifth graders of SDN 1 Cikalang and the sixth graders of SDN Karsanagara. 105 participants took part in this study. A survey was carried out to measure the value of a student's character. The results showed the development of their character lied mostly in the medium category. Scores for reading interest, creativity, curiosity, perseverance (diligence, heart strength, persistence), and devotion (religious) lied mostly in the low category.
\end{abstract}

Keywords: Rasch model; character; ethics; elementary school students

\section{Introduction}

The current phenomenon concerning degradation one's personality are mushrooming in Indonesia community. It is proven by the rise of student abuse cases. Cases of abuse do not only happen between students, but also happens between teachers and students. This is due to the loss of mutual respect and appreciation. Mubarok, Rusmana, Budiman \& Suryana (2019) argued that when individuals demonstrate disrespect attitude towards each other, it will adversely affect individuals with decreased learning outcomes, declining self-respect, and increased intimidation behavior to others in school as well as increasing violence or fostering unexpected immoral actions. The child's character can be influenced

* Corresponding author: Lutfi Nur, Email: lutfinur@upi.edu 
by the emotions of the child himself. It can even interfere the learning process if negative emotions arise in the child. Negative emotions such as feeling unhappy and disappointed then the learning process will experience obstacles (Nurillah, Zerlinda, Solehuddin \& Suryana, 2020). The influence of the family environment is huge, especially in children who are constantly faced with family suffering, bad care and high levels of conflict. They will grow and rise into children who will generally fail to manage their negative emotions and they can become aggressive adults (Saomah, Suryana \& Adzani, 2020). This is already in the realm of the destruction of the character of children at the age of the elementary school level. The prominent differences of children about learning, disciplined behavior, the way they dress, and the way they speak are clearly seen. These issues are caused by educational system such as zoning system (Yudha, Suryana \& Nabella, 2020).

Indonesia as a developing country has demonstrated its participation in the development of its people. Participation in the world of education also requires the application of policies to the school system to keep the developments in the education sector in line with global challenges (Sumintono, Said \& Mislan, 2012). The introduction of Information Technology amongst elementary school students has become a problem that needs to be confronted (Nur, Suherman \& Subarjah, 2019). Meanwhile, some researchers have indicated that sophisticated machinery can replace several professions in certain areas. However, character education cannot be taught by a lump of sophisticated machines. This means that a good character must be preserved by human beings as a hallmark of the Indonesian society. A good character can be imbued by teacher interactions with children.

The term character can also be considered as a value which is closely related to culture. Transformational value is an effort or action that is done to preserve or develop the cultural values (Wahyuni, Aji, Tias \& Sani, 2013). Good manners can be formed and inculcated through an educational process, which can be delivered by the teacher as a substitute of the parent's role at school. Character is etymologically derived from the Latin word "character" or the Greek word "kharassein", which means to mark it, or the French word "caracter", which means personality. In English, the word "character" means traits and roles. Character is seen as a personality trait which is associated with mental beings, namely mental conditions, and thought-forming processes. A person's character essentially consists of the qualities and relationships that exist between them. As for character traits, they are more than just a momentary state of mind, and just because someone shows certain thoughts does not mean that he has these underlying traits. A character adheres to the value of a person's behavior (Desstya, 2015).

This research has been carried out using the Rasch model approach through the Winstep program in order to assess the characters of elementary school students. Nurhudaya, Taufik, Yudha \& Suryana (2019) have used the Rasch model because it can provide solutions to the limitations of the classical model. The use of the Rasch's model approach can also determine the reliability of research instruments without depending on the sample, unlike the classic model where reliability depends on the sample, even though reliability in a test does not always have to depend on the sample (Van Der Ven \& Ellis, 2000). 
According to Sumintono \& Widhiarso (2015), the superiority of the Rasch's model compared to other model, especially the classical test theory, can be seen from its ability to predict missing data, based on individual response patterns. This advantage makes the statistical analysis results of Rasch model more accurate and authentic. It is certain that it the measurement of the character instrument of the primary school students is not employed based on the Rasch approach, it might cause research failure. Ardiyanti (2017) stated that the use of the Rasch model in instrument validation could result in more holistic information about the instrument and fulfill the measurement definitions. Therefore, the measurement of the character instrument of elementary school student uses the Rasch model approach to define the instrument measurements authentically and holistically.

Some research on character development has been done by Putri (2018) who discussed the character education of elementary school children in the digital age. Murniyetti, Engkizar and Anwar (2016) discussed the pattern of character education implementation in elementary school students. Supraptiningrum \& Agustini (2015) discussed how to build students ' character through school culture in elementary schools. Research on using computer-based Rasch applications has also been widely done. Ardiyanti (2017) used a Rasch model on the development of efficacy scale in career decision-making whereas Aziz (2015) applied the Rasch model for testing mental health measuring equipment in the workplace. Purba (2018) used the Rasch model to measure performance test instruments on basic subjects and electrical measurements and Makransky, Rogers, \& Creed (2015) used Rasch model to assess career decisions.

However, not much research has been done on the character measurement of elementary school students, especially by using the Rasch model approach through the Winstep program. This is evidenced by the fact that there is only one study by Misbach \& Sumintono (2014) on the measurements of character instrument validation. However, they focused on discussing students' perception of teachers' morals and character rather than discussing the character of individual students. The improvement of character must start from the teacher because teacher's discipline will affect to classroom management (Shih, Wu, Lai, \& Liao, 2015). There is no research focus on the character of elementary school students using Rasch modeling. Therefore, this article discusses the results of measuring student character with the Rasch model approach, through the Winstep program.

This research will answer the following questions:

1. How are the results of the distribution of instruments that reveal the character of students using the Rasch model?

2. Why Rasch Model can reveal the character of elementary school students? 


\section{Method}

This study employed a quantitative descriptive method to describe the character of elementary school students in Tasikmalaya, Indonesia. A purposive sampling technique was used in the study whereby a total of 105 high-grade elementary school students aged between 11 and 12 years old were taken as participants. This age range was selected because these students have the ability to think abstractly and logically. The opportunity to answer a question correctly depends on the ratio between one's ability and the difficulty level of the problem (Sumintono \& Widhiarso, 2014). Detail information of this student population is presented in Table 1.

Table 1. Selected Student Population

\begin{tabular}{lccc}
\hline \multirow{2}{*}{ School } & \multicolumn{2}{c}{ Population } & \multirow{2}{*}{ Total } \\
\cline { 2 - 3 } & $\mathbf{M}$ & $\mathbf{F}$ & \\
\hline SDN 1 Cikalang & 16 & 12 & 28 \\
\hline SDN Karsanagara & 35 & 42 & 77 \\
\hline Total & $\mathbf{5 1}$ & $\mathbf{5 4}$ & $\mathbf{1 0 5}$ \\
\hline
\end{tabular}

The instrument used in this research was in the form of a questionnaire which is used to reveal the character of students. Twenty-eight questions were prepared based on the aspects of good characters, including interest (strong desire), beliefs, confidence, perseverance (persistence), devout (religious), disciplined, honesty, tolerance, hard-working, creativity, independence, curiousness, respect, social care, peace, democratic, love for reading, and nationalism. These questions were presented in the form of statements and were answered based on a certain scale in accordance with the character of students.

The data obtained were processed using the Rasch model application. This model is developed to overcome problems that arise when using classical test theory in instrument analysis (Boone, 2016; Jackson, Draugalis, Slack, Zachry \& Agostino 2002). Thus, the Rasch model is seen as a measurement tool for mathematical analysis that can reveal the relationship between a person and the way he responds to the items in a given instrument (Jackson et al., 2002). The Rasch technique can be used to convert non-linear raw data on a linear scale which can then be evaluated using statistical parametric tests (Timofte \& Siminiciuc, 2018). Rasch model also has an interesting and easy advantage to apply at all scale formats. The Rasch model continues to develop not only for the analysis of dichotomous data, but also for polytomous data (Salzberger \& Sinkovics, 2006). Thus, the Rasch model is an excellent model for analyzing the validity of an instrument. However, there are important things to consider, for instance the number of participants and the number of parameters measured for each item. Having too many parameters with only few respondents may lead to the extraction of incorrect conclusions from the data (Timofte \& Siminiciuc, 2018). 


\section{Results}

Before analyzing the data further, it is worth measuring which instruments given to the respondent can be used to measure the character of elementary school students.

\section{Table 2. Undimensionality}

\begin{tabular}{|c|c|c|c|c|c|}
\hline & & Eigenvalue & Obser & rved & Expected \\
\hline Total raw variance in observations & $=$ & 41.9522 & $100.0 \%$ & & $100.0 \%$ \\
\hline Raw variance explained by measures & $=$ & 13.9522 & $33.3 \%$ & & $34.9 \%$ \\
\hline Raw variance explained by persons & $=$ & 4.4570 & $10.6 \%$ & & $11.1 \%$ \\
\hline Raw Variance explained by items & $=$ & 9.4952 & $22.6 \%$ & & $23.7 \%$ \\
\hline Raw unexplained variance (total) & $=$ & 28.0000 & $66.7 \%$ & $100.0 \%$ & $65.1 \%$ \\
\hline Unexplned variance in 1st contrast & $=$ & 3.3410 & $8.0 \%$ & $11.9 \%$ & \\
\hline Unexplned variance in 2 nd contrast & $=$ & 2.5825 & $6.2 \%$ & $9.2 \%$ & \\
\hline Unexplned variance in 3rd contrast & $=$ & 1.8051 & $4.3 \%$ & $6.4 \%$ & \\
\hline Unexplned variance in 4 th contrast & $=$ & 1.7771 & $4.2 \%$ & $6.3 \%$ & \\
\hline Unexplned variance in 5 th contrast & 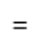 & 1.7392 & $4.1 \%$ & $6.2 \%$ & \\
\hline
\end{tabular}

Table 3. Person-Item Map

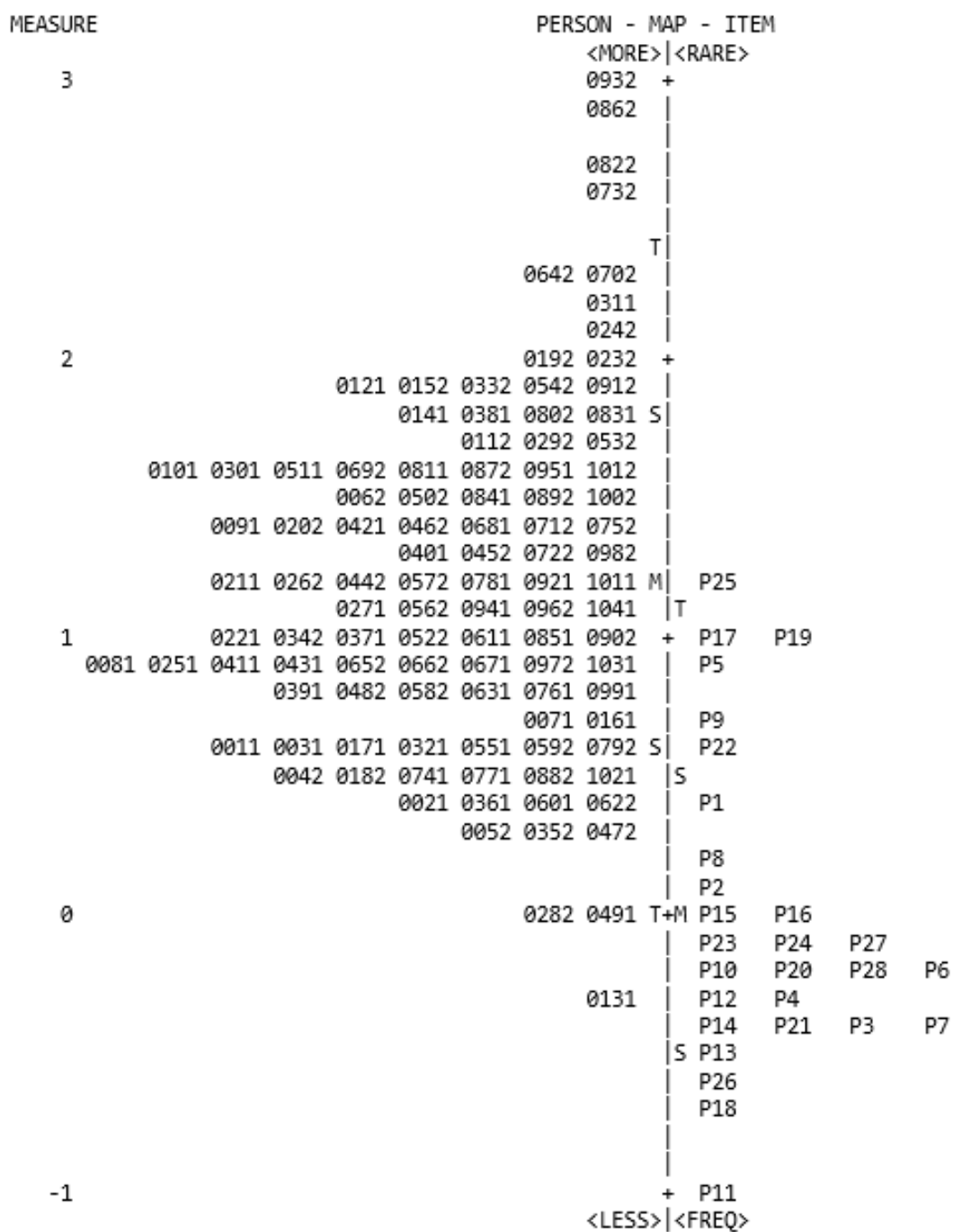


Table 2 shows that raw variance observe is $33.3 \%$, including adequate categories, while unexplained variance in the $1^{\text {st }}$ to the $5^{\text {th }}$ contrast of residuals are $11.9 \%$, $9.2 \%, 6.4 \%, 6.3 \%$ and $6.2 \%$. The spread of the respondent (the person) and the problem (item) can be seen in Table 3 .

Based on the Person-Item Map as shown in Table 3, we can see that the difficulty level of the items is spreads in the range 1 to 2 logits. A total of 27 items were positioned between $-2 \mathrm{SD}$ with $+2 \mathrm{SD}$, while one item, i.e., p25 number is above $+2 \mathrm{SD}$. The average level of ability of elementary school students (person) is above the standard difficulty level of the items.

Table 4. Difficulty level of items

\begin{tabular}{|c|c|c|c|c|c|c|c|c|c|c|c|c|c|}
\hline \multirow{2}{*}{$\begin{array}{l}\text { | ENTRY } \\
\text { | NUMBER }\end{array}$} & \multirow{2}{*}{$\begin{array}{l}\text { TOTAL } \\
\text { SCORE }\end{array}$} & \multirow{2}{*}{$\begin{array}{l}\text { TQTAL } \\
\text { COUNT }\end{array}$} & \multirow[b]{2}{*}{ MEASURE } & \multirow{2}{*}{$\begin{array}{l}\text { MODEL } \\
\text { S.E. }\end{array}$} & \multicolumn{2}{|c|}{ INFIT } & \multicolumn{2}{|c|}{ OUTFIT } & \multicolumn{2}{|c|}{ |PTMEASUR-AL } & \multirow{2}{*}{\begin{tabular}{|} 
|EXACT \\
| OBS\%
\end{tabular}} & \multirow{2}{*}{$\begin{array}{r}\text { MATCH } \\
\text { EXP\% }\end{array}$} & \multirow[b]{2}{*}{ Item } \\
\hline & & & & & |MNSQ & ZSTD & |MNSQ & ZSTD & ICORR. & EXP. I & & & \\
\hline 25 & 311 & 105 & 1.21 & .10 & .88 & -.96 & 1.90 & -.76 & .49 & $.51 \mid$ & 31.7 & 35.01 & P25 \\
\hline 17 & 335 & 105 & .98 & .10 & .98 & -.10 & 1.00 & .02 & .40 & .50 & 41.3 & $35.4 \mid$ & P17 \\
\hline 19 & 338 & 105 & .96 & .10 & .93 & -.53 & .91 & -.67 & .43 & .50 & 37.5 & 35.3 & P19 \\
\hline 5 & 346 & 105 & .88 & .10 & 1.94 & 6.16 & 2.06 & 6.63 & .26 & . 49 & 28.8 & $35.3 \mid$ & P5 \\
\hline 9 & 364 & 105 & .71 & .10 & 1.06 & .52 & 1.07 & .55 & .37 & .48 & 31.7 & $35.6 \mid$ & P9 \\
\hline 22 & 374 & 105 & .61 & .10 & 1.07 & .62 & 1.07 & .55 & .50 & .47 & 31.7 & 35.7 & P22 \\
\hline 1 & 397 & 105 & .37 & .10 & .82 & -1.42 & .87 & -.99 & .35 & $.45 \mid$ & 39.4 & $37.2 \mid$ & P1 \\
\hline 8 & 411 & 105 & .22 & .11 & 1.02 & .23 & 1.12 & .86 & .43 & $.43 \mid$ & 32.7 & $37.9 \mid$ & P8 \\
\hline 2 & 417 & 105 & .15 & .11 & .60 & -3.48 & .59 & -3.29 & .56 & .42 & 57.7 & $38.6 \mid$ & P2 \\
\hline 16 & 431 & 105 & -.02 & .11 & .94 & -.37 & .88 & -.74 & .50 & .40 & 45.2 & 41.9 & P16 \\
\hline 15 & 432 & 105 & -.04 & .11 & .87 & -.88 & .86 & -.90 & .33 & $.40 \mid$ & 49.0 & | & P15 \\
\hline 23 & 435 & 105 & -.08 & .12 & .95 & -.28 & .99 & .00 & .40 & $.40 \mid$ & 48.1 & $42.9 \mid$ & P23 \\
\hline 24 & 436 & 105 & -.09 & .12 & 1.17 & 1.14 & 1.43 & 2.46 & .46 & .40 & 48.1 & 42.9 & P24 \\
\hline 27 & 436 & 105 & -.09 & .12 & 1.15 & 1.06 & $\mid 1.09$ & .59 & .42 & $.40 \mid$ & 39.4 & $42.9 \mid$ & P27 \\
\hline 10 & 443 & 105 & -.19 & .12 & 11.03 & .27 & | .88 & -.75 & .54 & .38 & 47.1 & $44.9 \mid$ & P10 \\
\hline 28 & 443 & 105 & -.19 & .12 & 1.05 & .36 & 1.12 & .75 & .36 & .38 & 38.5 & $44.9 \mid$ & P28 \\
\hline 6 & 445 & 105 & -.21 & .12 & .95 & -.31 & .94 & -.33 & .41 & .38 & 50.0 & 45.6 & P6 \\
\hline 20 & 447 & 105 & -.24 & .12 & 1.41 & 2.47 & 1.35 & 1.92 & .35 & .38 & 46.2 & $45.7 \mid$ & P20 \\
\hline 12 & 448 & 105 & -.26 & .12 & .93 & -.44 & .84 & -.93 & .47 & $.37 \mid$ & 49.0 & $46.7 \mid$ & P12 \\
\hline 4 & 452 & 105 & -.32 & .12 & .93 & -.40 & .96 & -.16 & .44 & .37 & 53.8 & $48.1 \mid$ & P4 \\
\hline 7 & 454 & 105 & -.35 & .13 & 1.10 & .69 & .96 & -.18 & .40 & .36 & 54.8 & 48.3 & P7 \\
\hline 14 & 455 & 105 & -.37 & .13 & 11.03 & .23 & 11.04 & .28 & .38 & $.36 \mid$ & 50.0 & $48.4 \mid$ & P14 \\
\hline 21 & 456 & 105 & -.38 & .13 & .96 & -.23 & .91 & -.46 & .38 & .36 & 54.8 & $49.3 \mid$ & P21 \\
\hline 3 & 460 & 105 & -.45 & .13 & .76 & -1.55 & .77 & -1.30 & .31 & .35 & 55.8 & $50.9 \mid$ & P3 \\
\hline 13 & 464 & 105 & -.52 & .13 & .81 & -1.17 & .89 & -.54 & .43 & $.34 \mid$ & 68.3 & $52.0 \mid$ & P13 \\
\hline 26 & 470 & 105 & -.63 & .14 & 11.20 & 1.16 & 11.06 & .37 & .35 & $.33 \mid$ & 57.7 & $55.1 \mid$ & P26 \\
\hline 18 & 472 & 105 & -.67 & .14 & $\begin{array}{l}.77 \\
\end{array}$ & -1.37 & | .68 & -1.74 & .39 & .32 & 61.5 & 56.0 & P18 \\
\hline 11 & 486 & 105 & -1.00 & .16 & 1.20 & .99 & 1.07 & .36 & .32 & .28 & 67.3 & $66.0 \mid$ & P11 \\
\hline$-8-0$ & -- & & - & & & & & & & & 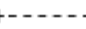 & & \\
\hline MEAN & 423.5 & 105.0 & .00 & .12 & 11.02 & .1 & $\mid 1.01$ & .1 & & & 47.0 & $44.3 \mid$ & \\
\hline P.SD & 45.8 & .0 & .54 & $.02 \mid$ & | .24 & 1.6 & | .26 & 1.7 & & & 10.6 & $7.4 \mid$ & \\
\hline
\end{tabular}

From Table 4, we can see that the standard deviation (SD) is 0.54 . If the elementary school value is combined with the mean value of logit $(0.0)$, then the grain difficulty level of the instrument (item) can be grouped into 3 categories: a very difficult category with provisions greater than 0.54, a difficult category with provisions between -0.54 and 0.54 and a very easy category with value less than -0.54. This form of research instrument used multiple choice questions (MCQs). The suitability of each option is shown in Table 5. 
Table 5. Rating Scale Diagnostic

\begin{tabular}{|c|c|c|c|c|c|c|c|c|c|c|}
\hline \multirow{2}{*}{$\begin{array}{l}\text { |CATEGORY } \\
\text { | LABEL }\end{array}$} & 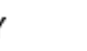 & \multicolumn{2}{|c|}{ OBSERVED | } & \multicolumn{2}{|c|}{ BSVD SAMPLE } & \multicolumn{2}{|c|}{ INFIT OUTFIT| } & ANDRICH & \multicolumn{2}{|l|}{ |CATEGORY| } \\
\hline & SCOR & E COUN & $\% \%$ & VRGE E & ХРЕCT| & MNSQ & MNSQII & THRESHOLD & MEASURE & \\
\hline & - & & & & & $\cdots$ & $-\cdots$ & -- & ---- & \\
\hline 1 & 1 & 66 & $2 \mid$ & .29 & $-.08 \mid$ & 1.42 & 1.57|| & NONE & $-2.50)$ & 1 \\
\hline 2 & 2 & 231 & 81 & .30 & .30 & .99 & 1.06|| & -1.15 & -.96 & 2 \\
\hline 3 & 3 & 509 & $17 \mid$ & .65 & .72 & .89 & .83|| & -.28 & .03 & 3 \\
\hline 4 & 4 & 867 & 29 & 1.15 & 1.16 & .96 & $.91 \mid$ & .41 & .97 & 4 \\
\hline 5 & 5 & 1267 & 431 & 1.64 & $1.61 \mid$ & 1.01 & 1.01|| & 1.03 & $2.42) \mid$ & 5 \\
\hline
\end{tabular}

The value of the Andrich Threshold in Table 5 shows that there is a match between the choice of answers from 1 to 5 as evidenced by an increase in the value of alternative answers from 1 to 5, with the following values: NONE, -1.15, -0.28, $0.03,0.97$, and 2.42. From Table 6 , it is concluded that there are 4 items that are biased: item $6(\mathrm{p}=0.445)$, item $8(\mathrm{p}=0.079)$, item $12(\mathrm{p}=0.047)$ and item $15(\mathrm{p}=$ $0.031)$.

Table 6. Item Bias Detection

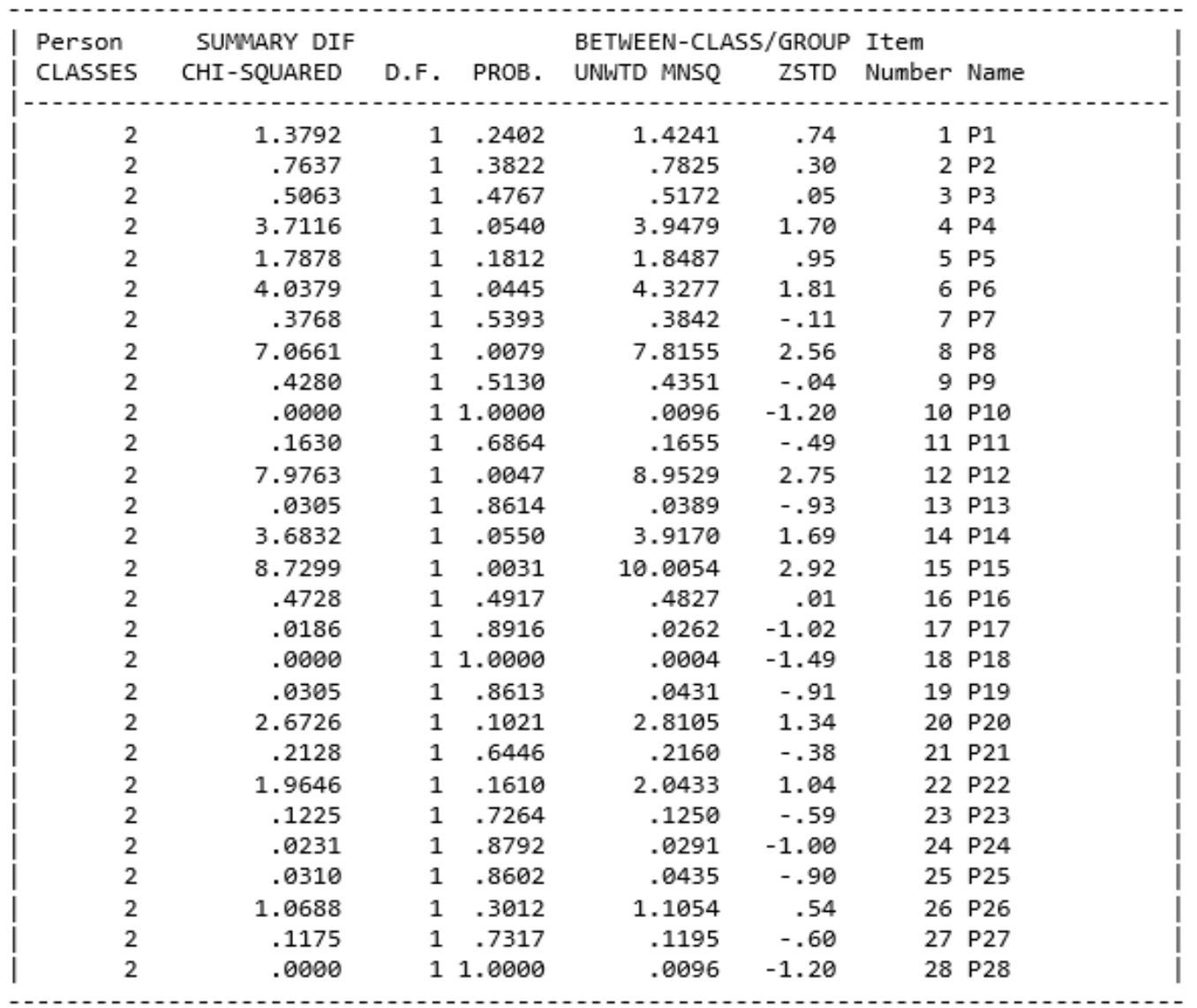

Based on Table 6 , it can be concluded that there are four items that are biased, i.e. item $6(p=0.445), 8(p=0.079), 12(p=0.047)$, and $15(p=0.031)$. Figure 1 (on page 9) shows that items 6 and 15 were the easiest to do for the sixth-graders at SDN Karsanagara, but tends to disadvantage the fifth-grade students at SDN 1 Cikalang. Items 8 and 12 were found to be the easiest ones to attempt by the fifthgraders at SDN 1 Cikalang, but they were found to be more difficult by the sixthgraders at SDN Karsanagara. 
Table 7. Person Measure

\begin{tabular}{|c|c|c|c|c|c|c|c|c|c|c|c|c|c|}
\hline \multirow{2}{*}{\begin{tabular}{|l} 
| ENTRY \\
NUMBER
\end{tabular}} & \multirow{2}{*}{$\begin{array}{l}\text { TOTAL } \\
\text { SCORE }\end{array}$} & \multirow{2}{*}{$\begin{array}{l}\text { IOTAL } \\
\text { COUNT }\end{array}$} & \multirow[b]{2}{*}{ MEASURE } & \multirow{2}{*}{$\begin{array}{l}\text { NOOEL } \\
\text { S.E. }\end{array}$} & & NFIT & our & TFIT & PTMEAS & R-AL| & EXACT & МATCH| & \\
\hline & & & & & INNSQ & ZSTD| & |NNSQ & ZSTD & cosi. & EXP.| & $005 x$ & EXPX| & Perso \\
\hline & & & & & & & & & & & & & \\
\hline 93 & 140 & 28 & 5.73 & 1.83 & MAXI & INUM ME & EASUR: & & .00 & .00 & 100.0 & 100.0 & 99322 \\
\hline 86 & 135 & 28 & 2.92 & .45 & .97 & $.12 \mid$ & | . .64 & $-.53 \mid$ & .50 & $.25 \mid$ & 85.7 & $83.2 \mid$ & 98622 \\
\hline 82 & 134 & 28 & 2.74 & .41 & 1.91 & 1.65 & 2.27 & 1.94 & .02 & $.27 \mid$ & 82.1 & $89.7 \mid$ & 98222 \\
\hline 73 & 133 & 28 & 2.58 & .38 & 2.32 & 2.29 & 1.38 & .85 & .31 & .29 & 75.0 & 75.5 & 97322 \\
\hline 64 & 131 & 28 & 2.33 & .34 & .93 & $-.84 \mid$ & 1.13 & $.42 \mid$ & -.13 & $.33 \mid$ & 53.6 & 70.11 & 96422 \\
\hline 70 & 131 & 28 & 2.33 & .34 & 1.19 & .57 & 1.27 & $.71 \mid$ & .17 & $.33 \mid$ & 67.9 & $70.1 \mid$ & 97922 \\
\hline 31 & 130 & 28 & 2.22 & .32 & 1.65 & 1.52 & 1.43 & 1.92 & .35 & $.34 \mid$ & 67.9 & 68.8 & 03112 \\
\hline 24 & 129 & 28 & 2.12 & .31 & .93 & $-.07 \mid$ & .75 & $-.52 \mid$ & .65 & $.35 \mid$ & 71.4 & $67.4 \mid$ & 92421 \\
\hline 19 & 128 & 28 & 2.03 & .30 & 1.54 & 1.49 & 1.52 & 1.26 & .15 & $.37 \mid$ & 53.6 & $65.0 \mid$ & 01921 \\
\hline 23 & 128 & 28 & 2.03 & .30 & 1.35 & .99| & .89 & $-.14 \mid$ & .68 & $.37 \mid$ & 82.1 & 65.0 & 02321 \\
\hline 12 & 127 & 28 & 1.94 & .29 & 1.06 & .29 & 1.18 & $.56 \mid$ & .58 & .38 & 67.9 & $62.6 \mid$ & 01211 \\
\hline 33 & 127 & 28 & 1.94 & .29 & 1.21 & .68 & .91 & $-.11 \mid$ & .49 & .38 & 67.9 & 62.6 & 93322 \\
\hline 15 & 126 & 28 & 1.86 & .28 & 1.94 & .22 & .94 & $-.03 \mid$ & .17 & . 39 & 50.0 & $60.3 \mid$ & 01521 \\
\hline 54 & 126 & 28 & 1.86 & .28 & .67 & -1.00 & .50 & -1.55 & .88 & . $39 \mid$ & 78.6 & $60.3 \mid$ & 05422 \\
\hline 91 & 126 & 28 & 1.86 & .28 & 1.29 & .90 & 1.28 & .81 & .40 & .39 & 64.3 & $60.3 \mid$ & 09122 \\
\hline 14 & 125 & 28 & 1.79 & .27 & 1.04 & .23 & 1.14 & .49 & .58 & .49 & 64.3 & 59.2 & 01411 \\
\hline 38 & 125 & 28 & 1.79 & .27 & .99 & .07 & .87 & $-.26 \mid$ & .64 & $.49 \mid$ & 64.3 & 59.2 & 03812 \\
\hline 80 & 125 & 28 & 1.79 & .27 & .95 & -.03 & .77 & -.56 & .70 & .49 & 75.0 & 59.2 & 98022 \\
\hline 83 & 125 & 28 & 1.79 & .27 & 1.06 & .28 & 1.08 & $.35 \mid$ & .58 & $.40 \mid$ & 64.3 & 59.2 & 08312 \\
\hline 11 & 124 & 28 & 1.72 & .26 & 1.02 & .16 & .88 & -.23 & .23 & .41 & 42.9 & 58.0 & 01121 \\
\hline 29 & 124 & 28 & 1.72 & .26 & |1.11 & $.44 \mid$ & .94 & $-.07 \mid$ & .73 & $.41 \mid$ & 71.4 & 58.0 & 02922 \\
\hline 53 & 124 & 28 & 1.72 & .26 & .74 & $-.77 \mid$ & .69 & -.88 & .61 & $.41 \mid$ & 53.6 & 58.0 & 05322 \\
\hline 10 & 123 & 28 & 1.65 & .26 & 1.07 & $.33 \mid$ & 1.22 & .72 & .52 & $.42 \mid$ & 57.1 & 54.8 & 01011 \\
\hline 30 & 123 & 28 & 1.65 & .26 & 1.42 & 1.28 & .96 & -.01 & .83 & .42 & 67.9 & 54.8 & 03012 \\
\hline 95 & 123 & 28 & 1.65 & .26 & .59 & $-1.43 \mid$ & .52 & $-1.57 \mid$ & .55 & $.42 \mid$ & 67.9 & 54.8 & 09512 \\
\hline 51 & 122 & 28 & 1.58 & .25 & .56 & -1.59 & .61 & -1.25 & .34 & .42 & 67.9 & $50.9 \mid$ & 05112 \\
\hline 69 & 122 & 28 & 1.58 & .25 & .91 & -.18 & 1.00 & .11| & .36 & $.42 \mid$ & 32.1 & 50.9 & 96922 \\
\hline 81 & 122 & 28 & 1.58 & .25 & .43 & -2.26 & .48 & -1.82 & .66 & .42 & 53.6 & 50.9 & 98112 \\
\hline 87 & 122 & 28 & 1.58 & .25 & .91 & -.21 & 1.01 & .15 & .42 & $.42 \mid$ & 50.0 & 50.9 & 08722 \\
\hline 102 & 122 & 28 & 1.58 & .25 & .80 & -.60 & .67 & -1.00 & .64 & .42 & 75.0 & $50.9 \mid$ & 10222 \\
\hline 50 & 121 & 28 & 1.52 & .24 & .78 & $-.67 \mid$ & .74 & $-.78 \mid$ & .56 & $.43 \mid$ & 35.7 & $48.1 \mid$ & 95022 \\
\hline 89 & 121 & 28 & 1.52 & .24 & .95 & -.08 & .80 & $-.56 \mid$ & .58 & $.43 \mid$ & 42.9 & 48.11 & 08922 \\
\hline 6 & 120 & 28 & 1.46 & .24 & 1.67 & 1.97 & 1.99 & 2.51 & .19 & .44 & 50.0 & 45.5 & 00621 \\
\hline 84 & 120 & 28 & 1.46 & .24 & | .90 & -.26 & .79 & $-.58 \mid$ & .63 & $.44 \mid$ & 39.3 & 45.5 & 08412 \\
\hline 100 & 120 & 28 & 1.46 & .24 & 1.16 & .62 & .97 & $.00 \mid$ & .64 & $.44 \mid$ & 35.7 & 45.5 & 10022 \\
\hline 9 & 119 & 28 & 1.41 & .24 & .95 & -.07 & 1.13 & .50 & .50 & .44 & 42.9 & 44.1 & 00911 \\
\hline 20 & 119 & 28 & 1.41 & .24 & .79 & $-.67 \mid$ & .86 & $-.35 \mid$ & .65 & $.44 \mid$ & 42.9 & $44.1 \mid$ & 02021 \\
\hline 42 & 119 & 28 & 1.41 & .24 & 1.31 & 1.86 & 1.23 & $.80 \mid$ & .43 & $.44 \mid$ & 35.7 & 44.11 & 04212 \\
\hline 68 & 119 & 28 & 1.41 & .24 & 1.78 & 2.26 & 1.73 & 2.03 & .34 & $.44 \mid$ & 35.7 & 44.1 & 96812 \\
\hline 46 & 118 & 28 & 1.35 & .23 & 1.49 & 1.57 & 1.86 & 2.34 & -.11 & .45 & 53.6 & 40.71 & 04622 \\
\hline 71 & 118 & 28 & 1.35 & .23 & 1.32 & 1.11 & 1.09 & .37 & .18 & .45 & 42.9 & 40.7 & 97122 \\
\hline 75 & 118 & 28 & 1.35 & .23 & | .96 & -.03 & | .79 & $-.63 \mid$ & .30 & $.45 \mid$ & 42.9 & $40.7 \mid$ & 97522 \\
\hline 49 & 117 & 28 & 1.30 & .23 & 1.19 & .73 & 1.08 & .37 & .59 & .45 & 35.7 & 39.7 & 04012 \\
\hline 45 & 117 & 28 & 1.30 & .23 & .58 & -1.65 & .55 & -1.69 & .16 & .45 & 64.3 & 39.7 & 94522 \\
\hline 72 & 117 & 28 & 1.30 & .23 & | .69 & $-1.14 \mid$ & | .73 & $-.91 \mid$ & .57 & $.45 \mid$ & 28.6 & $39.7 \mid$ & 07222 \\
\hline 98 & 117 & 28 & 1.30 & .23 & 1.48 & 1.58 & 1.43 & $1.37 \mid$ & .26 & $.45 \mid$ & 32.1 & 39.7 & 99822 \\
\hline 44 & 116 & 28 & 1.25 & .22 & 1.94 & .23 & .90 & -.25 & .76 & $.46 \mid$ & 35.7 & 38.8 & 04422 \\
\hline 57 & 116 & 28 & 1.25 & .22 & .97 & -.02 & 1.00 & $.08 \mid$ & .36 & $.46 \mid$ & 57.1 & $38.8 \mid$ & 05722 \\
\hline 21 & 115 & 28 & 1.20 & .22 & .95 & -.08 & .94 & $-.11 \mid$ & .29 & $.46 \mid$ & 50.0 & 37.8 & 02111 \\
\hline 26 & 115 & 28 & 1.20 & .22 & .85 & -.48 & .93 & $-.14 \mid$ & .32 & .46 & 46.4 & 37.8 & 92621 \\
\hline 78 & 114 & 28 & 1.15 & .22 & 1.25 & .94 & 1.35 & 1.20 & .39 & $.47 \mid$ & 42.9 & $37.9 \mid$ & 07812 \\
\hline $\begin{array}{r}92 \\
101\end{array}$ & $\begin{array}{l}114 \\
114\end{array}$ & $\begin{array}{l}28 \\
28\end{array}$ & $\begin{array}{l}1.15 \\
1.15\end{array}$ & $\begin{array}{l}.22 \\
.22\end{array}$ & $\begin{array}{l}.98 \\
.71\end{array}$ & $\begin{array}{r}.021 \\
-1.08\end{array}$ & $\begin{array}{l}.95 \\
.77\end{array}$ & $\begin{array}{l}-.091 \\
-.78\end{array}$ & $\begin{array}{l}.68 \\
.41\end{array}$ & $\begin{array}{l}.47 \mid \\
.47 \mid\end{array}$ & $\begin{array}{l}28.6 \\
42.9\end{array}$ & $\begin{array}{l}37.9 \\
37.9\end{array}$ & $\begin{array}{l}99212 \\
10112\end{array}$ \\
\hline 27 & 113 & 28 & 1.10 & .22 & .85 & -.48 & .86 & -.44 & .48 & .47 & 39.3 & 38.0 & 02711 \\
\hline 94 & 113 & 28 & 1.10 & .22 & \begin{tabular}{|l|l} 
& .60
\end{tabular} & -1.64 & .62 & -1.49 & .69 & .47 & 39.3 & 38.0 & 09412 \\
\hline 96 & 113 & 28 & 1.10 & .22 & $\begin{array}{l}.76 \\
\end{array}$ & -.89 & .76 & -.82 & .70 & .47 & 32.1 & 38.0 & 09622 \\
\hline 56 & 112 & 28 & 1.06 & .21 & 1.31 & 1.14 & 1.31 & $1.13 \mid$ & .41 & .48 & 42.9 & 37.0 & 05622 \\
\hline 105 & 112 & 28 & 1.06 & .21 & 1.29 & 1.09 & 1.24 & .90 & .56 & .48 & 17.9 & 37.0 & 10512 \\
\hline 34 & 111 & 28 & 1.01 & .21 & . .58 & -1.79 & \begin{tabular}{|l} 
| \\
\end{tabular} & -1.41 & .49 & .48 & 57.1 & 36.2 & 03422 \\
\hline 52 & 111 & 28 & 1.01 & .21 & 1.38 & 1.39 & 1.38 & $1.33 \mid$ & .51 & .48 & 28.6 & 36.2 & 95222 \\
\hline 90 & 111 & 28 & 1.01 & .21 & 1.65 & 2.15 & 1.54 & 1.79 & .64 & .48 & 10.7 & 36.2 & 09022 \\
\hline 22 & 110 & 28 & .97 & .21 & | .83 & -.62 & | .81 & -.67 & .65 & .48 & 39.3 & 36.3 & 02211 \\
\hline 37 & 110 & 28 & .97 & .21 & |1.11 & $.51 \mid$ & 1.10 & $.44 \mid$ & .45 & $.48 \mid$ & 25.0 & $36.3 \mid$ & 03712 \\
\hline 61 & 110 & 28 & .97 & .21 & \begin{tabular}{|l}
.76 \\
\end{tabular} & -.91 & \begin{tabular}{|l}
.79 \\
\end{tabular} & $-.73 \mid$ & .52 & .48 & 35.7 & 36.31 & 06112 \\
\hline 85 & 110 & 28 & .97 & .21 & .88 & -.38 & .77 & -.85 & .59 & .48 & 50.0 & 36.3 & 08512 \\
\hline 25 & 109 & 28 & .93 & .21 & 1.93 & 2.93 & 1.69 & 2.25 & .49 & .48 & 21.4 & 36.4 & 02511 \\
\hline 43 & 109 & 28 & .93 & .21 & .80 & -.77 & . .83 & -.59 & .43 & .48 & 39.3 & 36.4 & 04312 \\
\hline 66 & 109 & 28 & .93 & .21 & 1.34 & 1.27 & 1.36 & 1.32 & .54 & .48 & 21.4 & 36.4 & 96622 \\
\hline 67 & 109 & 28 & .93 & .21 & \begin{tabular}{|l|l|} 
&
\end{tabular} & -.06 & | .99 & .04 & .68 & .48 & 35.7 & $36.4 \mid$ & 06712 \\
\hline 97 & 109 & 28 & .93 & .21 & .56 & -1.97 & .56 & -1.87 & .60 & .48 & 50.0 & 36.4 & 09722 \\
\hline 104 & 109 & 28 & .93 & .21 & .75 & -1.00 & .79 & -.77 & .55 & .48 & 35.7 & 36.4 & 18412 \\
\hline 8 & 108 & 28 & .88 & .20 & \begin{tabular}{|l|} 
\\
\end{tabular} & -.27 & | . .80 & -.72 & .66 & .49 & 46.4 & 36.5 & 00811 \\
\hline
\end{tabular}




\begin{tabular}{|c|c|c|c|c|c|c|c|c|c|c|c|c|c|}
\hline 41 & 108 & 28 & .88 & .20 & .87 & -.43 & .83 & -.58 & .49 & .49 & 46.4 & 36.5 & 94112 \\
\hline 65 & 108 & 28 & .88 & .20 & .83 & $-.61 \mid$ & .75 & $-.94 \mid$ & .43 & $.49 \mid$ & 46.4 & $36.5 \mid$ & 96522 \\
\hline 58 & 107 & 28 & .84 & .20 & .89 & -.36 & .92 & -.25 & .51 & .49 & 42.9 & 36.5 & 95822 \\
\hline 63 & 107 & 28 & .84 & .20 & .63 & -1.62 & .56 & -1.92 & .31 & .49 & 60.7 & 36.5 & 96312 \\
\hline 39 & 186 & 28 & .80 & .20 & 1.24 & .98 & 1.22 & .89 & .72 & .49 & 21.4 & $36.5 \mid$ & 03912 \\
\hline 76 & 186 & 28 & .80 & .20 & .94 & -.15 & .86 & -.49 & .00 & .49 & 60.7 & 36.5 & 07612 \\
\hline 48 & 105 & 28 & .76 & .20 & .28 & -4.95 & 19 & $-4.95 \mid$ & .76 & $.49 \mid$ & 78.6 & $36.6 \mid$ & 94822 \\
\hline 99 & 105 & 28 & .76 & $.20 \mid$ & 1.34 & 1.33 & 1.45 & $1.64 \mid$ & .23 & $.49 \mid$ & 28.6 & $36.6 \mid$ & 09912 \\
\hline 7 & 103 & 28 & .68 & .20 & 1.19 & .82 & 1.19 & .79 & .27 & .49 & 42.9 & $35.4 \mid$ & 90711 \\
\hline 16 & 103 & 28 & .68 & $.20 \mid$ & .31 & -3.83 & .31 & -3.80 & .44 & .49 & 71.4 & $35.4 \mid$ & 01611 \\
\hline 3 & 102 & 28 & .65 & .19| & .38 & -3.32 & .38 & -3.22 & .68 & $.50 \mid$ & 60.7 & $35.4 \mid$ & 80311 \\
\hline 17 & 102 & 28 & .65 & .19 & .26 & -4.42 & .26 & -4.34 & .55 & .50 & 75.0 & 35.4 & 01711 \\
\hline 59 & 102 & 28 & .65 & .19| & 1.13 & .60 & 1.11 & .49 & .52 & .50 & 35.7 & 35.4 & 05922 \\
\hline 79 & 102 & 28 & .65 & .19| & .54 & -2.18 & .55 & -2.09 & .59 & .50 & 46.4 & 35.4 & 07922 \\
\hline 32 & 101 & 28 & .61 & .19 & .52 & -2.33 & .51 & -2.35 & .30 & .50 & 50.0 & 35.3 & 03212 \\
\hline 1 & 100 & 28 & .57 & .19| & .82 & -.71 & .88 & -.78 & .50 & $.50 \mid$ & 42.9 & 35.2 & 80111 \\
\hline 55 & 100 & 28 & .57 & .19| & |1.94 & 3.17 & |1.96 & $3.17 \mid$ & .01 & $.50 \mid$ & 25.8 & 35.2 & 05512 \\
\hline 18 & 99 & 28 & .53 & .19| & .69 & -1.40 & .66 & -1.54 & .63 & .50 & 46.4 & 35.3 & 01821 \\
\hline 77 & 98 & 28 & .50 & .19| & .96 & -.09 & .94 & $-.20 \mid$ & .23 & .50 & 35.7 & $35.4 \mid$ & 07712 \\
\hline 88 & 98 & 28 & .50 & .19| & 11.00 & . 89 & 1.00 & $.86 \mid$ & .23 & $.50 \mid$ & 28.6 & $35.4 \mid$ & 98822 \\
\hline 4 & 97 & 28 & .46 & .19 & 1.09 & .44 & 1.94 & .26 & .29 & .50 & 42.9 & $35.3 \mid$ & 00421 \\
\hline 74 & 97 & 28 & .46 & .19| & .84 & -.66 & .79 & -.86 & .45 & .50 & 39.3 & 35.3 & 07412 \\
\hline 103 & 97 & 28 & .46 & .19| & 1.60 & 2.22 & |1.65 & $2.35 \mid$ & .37 & $.50 \mid$ & 39.3 & $35.3 \mid$ & 10312 \\
\hline 36 & 95 & 28 & .39 & .19| & .55 & -2.25 & .53 & -2.35 & .42 & .50 & 42.9 & 35.1 & 83612 \\
\hline 60 & 95 & 28 & .39 & .19| & 1.38 & 1.53 & 1.48 & 1.84 & .47 & .50 & 28.6 & 35.1 & 06012 \\
\hline 2 & 94 & 28 & .35 & .19| & 1.93 & 3.21 & |1.93 & 3.19 & .45 & .50 & 25.8 & 34.9 & 00211 \\
\hline 62 & 94 & 28 & .35 & .19 & 1.11 & .52 & 1.12 & .57 & .47 & .50 & 35.7 & 34.9 & 96222 \\
\hline 47 & 93 & 28 & .32 & .19| & 1.15 & .70 & 1.14 & $.64 \mid$ & .35 & .50 & 28.6 & 34.9 & 94722 \\
\hline 5 & 92 & 28 & .28 & .19| & .78 & -.95 & .77 & - & .58 & .50 & 35.7 & 34.9 & 00521 \\
\hline 35 & 92 & 28 & .28 & .19| & .78 & -.95 & .79 & $-.90 \mid$ & .32 & .50 & 35.7 & 34.9 & 83522 \\
\hline 28 & 85 & 28 & .84 & .18 & $\mid 1.22$ & .98 & 1.18 & .79| & .45 & .49 & 46.4 & 35.01 & 02821 \\
\hline 49 & 85 & 28 & .84 & .18 & 3.00 & 5.83 & 3.98 & 5.94 & .94 & .49 & 14.3 & 35.8 & 94912 \\
\hline 13 & 76 & 28 & -.26 & .19| & .78 & $-.93 \mid$ & .80 & -.82 & .48 & $.47 \mid$ & 25.8 & 35.2 & 01311 \\
\hline & & 8.8 & .22 & .25 & 1.94 & 5 & L.8. & $\because$ & & & 47. & 4. & \\
\hline & 12.2 & .0 & .74 & $.16 \mid$ & .43 & 1.5 & | . .43 & $1.5 \mid$ & & & 16.8 & 11.9 & \\
\hline
\end{tabular}

Table 7 shows the Person Measure or the level of ability of students in working with the character instrument of elementary school students. The standard deviation (SD) of their abilities is 0.74 . This value if combined with the mean of logit values (1.22), the individual ability level of students regarding their character can be grouped into three categories: a high ability category with the provision of a value greater than $1.22+0.74=1.96$, a medium ability category with provisions between $0.48(1.22-0.74)$ and $1.96(1.22+0.74)$ and a low level ability category with value less than 0.48 . Thus, from the 105 students who were surveyed, there are 10 high ability students, 82 with moderate ability, and 13 with low ability.

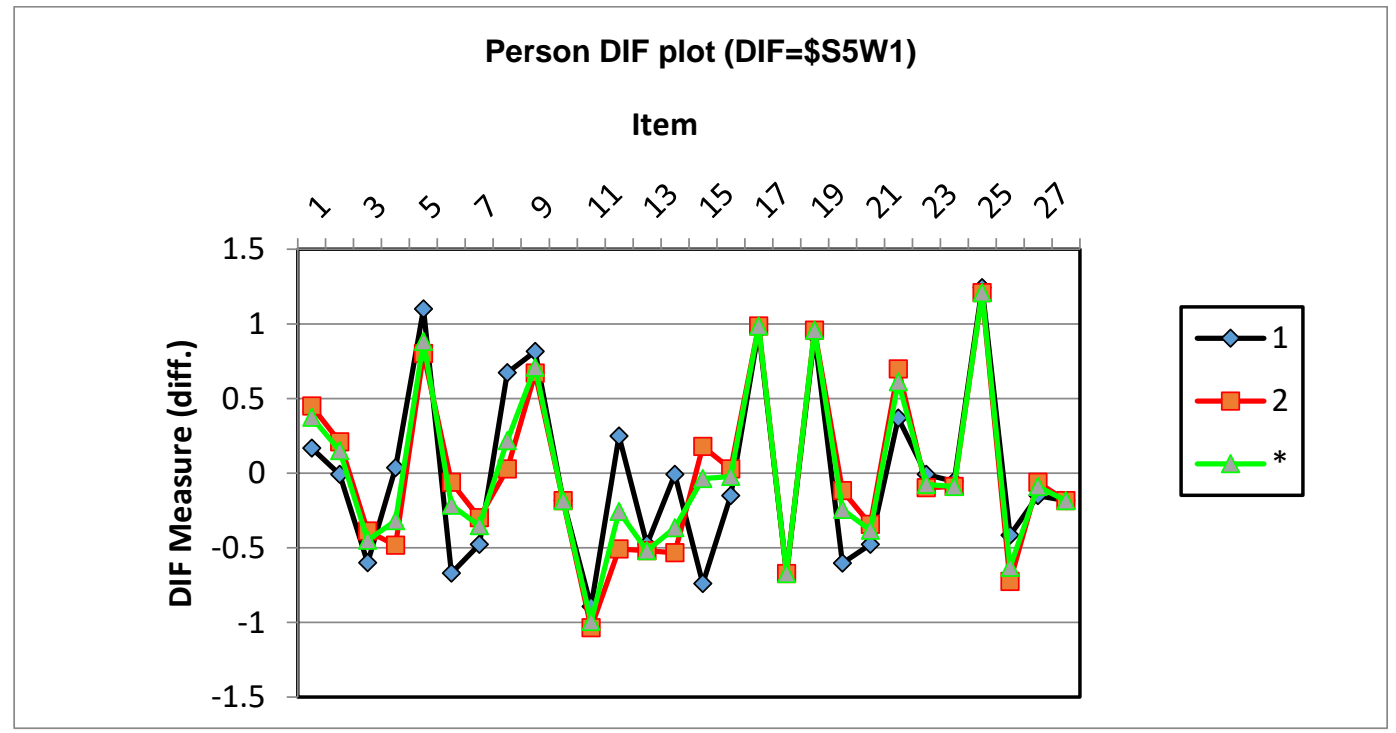

Figure 1. Item Bias Detection 
Table 8. Person Fit Order

\begin{tabular}{|c|c|c|c|c|c|c|c|c|c|c|c|c|c|}
\hline & TOTAL & JoTAL & & MODEL| & & FIT & OUT & FFIT & |PTMEAS & IR-ALI & EXACT & МATCH| & \\
\hline VBER & SCORE & COUNT & MEASURE & S.E. IN & NWSQ & ZSTD|M & MNSQ & ZSTOIf & I CORR. & EXP.I & OBS\% & EXPX| & Perso \\
\hline 49 & 85 & 28 & .84 & $.18 \mid 3$ & 3.60 & $5.83 \mid 3$ & 3.68 & $5.94 \mid$ & |A. .84 & $.49 \mid$ & 14.3 & 35.01 & 64912 \\
\hline 73 & 133 & 28 & 2.58 & $.38 \mid 2$ & 2.32 & $2.29 \mid 1$ & 1.38 & .85 & B .31 & .29 & 75.6 & 75.51 & 67322 \\
\hline 82 & 134 & 28 & 2.74 & $.41 \mid 1$ & 1.91 & $1.65 \mid 2$ & 2.27 & $1.94 \mid c$ & |c .02 & .27 & 82.1 & $80.7 \mid$ & 68222 \\
\hline 6 & 120 & 28 & 1.46 & $.24 \mid 1$ & 1.67 & $1.97 \mid 1$ & 1.99 & $2.51 \mid 0$ & D. .19 & $.44 \mid$ & 56.8 & $45.5 \mid$ & 60621 \\
\hline 55 & 100 & 28 & .57 & .19|1 & 1.94 & $3.17 \mid 1$ & 1.96 & $3.17 \mid$ & |E..01 & .58 & 25.6 & 35.21 & 65512 \\
\hline 2 & 94 & 28 & .35 & .19 & 1.93 & $3.21 \mid 1$ & 1.93 & $3.19 \mid$ & |F .45 & .58 & 25.6 & $34.9 \mid$ & 60211 \\
\hline 25 & 109 & 28 & .93 & $.21 \mid 1$ & 1.93 & $2.93 \mid 1$ & 1.69 & $2.25 \mid c$ & |G .49 & $.48 \mid$ & 21.4 & $36.4 \mid$ & 02511 \\
\hline 46 & 118 & 28 & 1.35 & $.23 \mid 1$ & 1.49 & $1.57 \mid 1$ & 1.86 & $2.34 \mid 1$ & |H-.11 & $.45 \mid$ & 53.6 & 40.71 & 64622 \\
\hline 68 & 119 & 28 & 1.41 & $.24 \mid 1$ & 1.78 & $2.26 \mid 1$ & 1.73 & 2.03 & | I . .34 & $.44 \mid$ & 35.7 & $44.1 \mid$ & 66812 \\
\hline 31 & 130 & 28 & 2.22 & $.32 \mid 1$ & 1.65 & $1.52 \mid 1$ & 1.43 & $1.82 \mid$ & |]. 35. & $.34 \mid$ & 67.9 & $68.8 \mid$ & 03112 \\
\hline 96 & 111 & 28 & 1.01 & $.21 \mid 1$ & 1.65 & $2.15 \mid 1$ & 1.54 & $1.79 \mid \mathrm{r}$ & |K. .64 & $.48 \mid$ & 10.7 & 36.21 & 69822 \\
\hline 103 & 97 & 28 & .46 & .19 & 1.60 & $2.22 \mid 1$ & 1.65 & $2.35 \mid$ & |L. .37 & .58 & 39.3 & 35.31 & 10212 \\
\hline 19 & 128 & 28 & 2.83 & .30 & 1.54 & $1.40 \mid 1$ & 1.52 & $1.26 \mid \mathrm{M}$ & |M.15 & $.37 \mid$ & 53.6 & 65.01 & 61921 \\
\hline 60 & 95 & 28 & .39 & .19|1 & 1.38 & $1.53 \mid 1$ & 1.48 & 1.84 & |N . .47 & .58 & 28.6 & $35.1 \mid$ & 66012 \\
\hline 98 & 117 & 28 & 1.30 & $.23 \mid 1$ & 1.48 & $1.58 \mid 1$ & 1.43 & $1.37 \mid c$ & 10.26 & $.45 \mid$ & 32.1 & $39.7 \mid$ & 69822 \\
\hline 99 & 105 & 28 & .76 & .2811 & 1.34 & $1.33 \mid 1$ & 1.45 & $1.64 \mid \mathrm{F}$ & |P.23 & .49| & 28.6 & $36.6 \mid$ & 69912 \\
\hline 38 & 123 & 28 & 1.65 & .26 & 1.42 & 1.28 & |.96 & $-.01 \mid$ & |Q .83 & $.42 \mid$ & 67.9 & $54.8 \mid$ & 03812 \\
\hline 52 & 111 & 28 & 1.01 & $.21 \mid 1$ & 1.38 & $1.39 \mid 1$ & 1.38 & $1.33 \mid \mathrm{f}$ & |R . . 51 & .48 & 28.6 & 36.21 & 65222 \\
\hline 66 & 109 & 28 & .93 & $.21 \mid 1$ & 1.34 & $1.27 \mid 1$ & 1.36 & $1.32 \mid \mathrm{s}$ & I5 .54 & .48 & 21.4 & $36.4 \mid$ & 66622 \\
\hline 23 & 128 & 28 & 2.03 & $.30 \mid 1$ & 1.35 & .99| & . .89 & $-.14 \mid 7$ & |T .68 & $.37 \mid$ & 82.1 & 65.0 이 & 02321 \\
\hline 78 & 114 & 28 & 1.15 & .2211 & 1.25 & $.94 \mid 1$ & 1.35 & $1.28 \mid \mathrm{L}$ & |U .39 & $.47 \mid$ & 42.9 & $37.9 \mid$ & 67812 \\
\hline 71 & 118 & 28 & 1.35 & $.23 \mid 1$ & 1.32 & $1.11 \mid 1$ & 1.69 & $.371 \mathrm{C}$ & V .18 & .45 & 42.9 & $48.7 \mid$ & 67122 \\
\hline 42 & 119 & 28 & 1.41 & .24 & 1.31 & $1.86 \mid$ & 1.23 & $.80 \mid \mathrm{r}$ & |W . .43 & $.44 \mid$ & 35.7 & $44.1 \mid$ & 64212 \\
\hline 56 & 112 & 28 & 1.86 & .21 & 1.31 & 1.14 & |1. 31 & $1.13 \mid \gamma$ & |X . .41 & .48 & 42.9 & 37.81 & 65622 \\
\hline 91 & 126 & 28 & 1.86 & .28 & 1.29 & $.90 \mid 1$ & 1.28 & $.81 \mid$ & |Y . .46 & .39| & 64.3 & 60.3 & 69122 \\
\hline 105 & 112 & 28 & 1.86 & .21 & 1.29 & $1.09 \mid$ & 1.24 & $.90 \mid 2$ & |z .56 & .48 & 17.9 & 37.01 & 16412 \\
\hline 76 & 131 & 28 & 2.33 & $.34 \mid 1$ & 1.19 & $.57 \mid 1$ & 1.27 & .71| & .17 & .33| & 67.9 & $70.1 \mid$ & 67022 \\
\hline 39 & 106 & 28 & .80 & .201 & 1.24 & $.98 \mid$ & 1.22 & . 89 & .72 & .49 & 21.4 & 36.51 & 03912 \\
\hline 16 & 123 & 28 & 1.65 & .26 & 1.87 & $.33 \mid$ & 1.22 & .721 & .52 & $.42 \mid$ & 57.1 & 54.81 & 01011 \\
\hline 28 & 85 & 28 & .84 & $.18 \mid$ & 1.22 & .98 & 1.18 & $.79 \mid$ & .45 & .49 & 46.4 & 35.81 & 02821 \\
\hline 33 & 127 & 28 & 1.94 & .29 & 1.21 & .68 & | .91 & $-.11 \mid$ & .49 & .38 & 67.9 & $62.6 \mid$ & 63322 \\
\hline 64 & 131 & 28 & 2.33 & $.34 \mid$ & .93 & $-.04 \mid 1$ & 1.13 & $.42 \mid$ & -.13 & $.33 \mid$ & 53.6 & $70.1 \mid$ & 66422 \\
\hline 86 & 135 & 28 & 2.92 & $.45 \mid$ & .97 & $.12 \mid$ & | .64 & $-.53 \mid$ & .50 & $.25 \mid$ & 85.7 & 83.21 & 08622 \\
\hline & BETTER & FITTING & NOT SHOWN & & $\ldots$ & $\cdots+$ & $+\cdots$ & ....t & & & & & \\
\hline 75 & 118 & 28 & 1.35 & .23 & .96 & -.03 & |.79 & $-.63 \mid$ & .30 & $.45 \mid$ & 42.9 & 46.7| & 67522 \\
\hline 80 & 125 & 28 & 1.79 & $.27 \mid$ & .95 & $-.03 \mid$ & .77 & $-.56 \mid$ & .70 & .48 & 75.6 & 59.2 & 68022 \\
\hline 89 & 121 & 28 & 1.52 & $.24 \mid$ & .95 & $-.98 \mid$ & |.89 & $-.56 \mid$ & .58 & $.43 \mid$ & 42.9 & $48.1 \mid$ & 08922 \\
\hline 76 & 186 & 28 & .80 & .280 & .94 & -.15 & .86 & $-.49 \mid$ & .80 & .49| & 60.7 & $36.5 \mid$ & 67612 \\
\hline 24 & 129 & 28 & 2.12 & $.31 \mid$ & .93 & $-.07 \mid$ & .75 & $-.52 \mid$ & .65 & $.35 \mid$ & 71.4 & $67.4 \mid$ & 02421 \\
\hline 84 & 120 & 28 & 1.46 & $.24 \mid$ & .96 & $-.26 \mid$ & .79 & $-.58 \mid$ & .63 & $.44 \mid$ & 39.3 & 45.5 & 68412 \\
\hline 85 & 110 & 28 & .97 & $.21 \mid$ & .88 & -.38 & .77 & -.85 & .59 & .48 & 50.6 & $36.3 \mid$ & 68512 \\
\hline 26 & 119 & 28 & 1.41 & .24 & .79 & $-.67 \mid$ & .86 & $-.35 \mid$ & .65 & $.44 \mid$ & 42.9 & $44.1 \mid$ & 02021 \\
\hline 74 & 97 & 28 & .46 & .19| & .84 & -.66 & .79 & $-.86 \mid$ & .45 & .50 & 39.3 & 35.31 & 67412 \\
\hline 43 & 109 & 28 & .93 & .21 & .88 & $-.77 \mid$ & .83 & -.59 & .43 & .48 & 39.3 & 36.4 & 64312 \\
\hline 65 & 108 & 28 & .88 & .20 & .83 & $-.61 \mid$ & .75 & $-.94 \mid$ & .43 & .49 & 46.4 & 36.5 & 66522 \\
\hline 13 & 76 & 28 & -.26 & .19| & .78 & $-.93 \mid$ & .80 & $-.82 \mid$ & .40 & $.47 \mid$ & 25.6 & 35.2 & 01311 \\
\hline 102 & 122 & 28 & 1.58 & $.25 \mid$ & .80 & $-.60 \mid$ & .67 & $-1.60 \mid$ & .64 & $.42 \mid$ & 75.6 & $50.9 \mid$ & 16122 \\
\hline 35 & 92 & 28 & .28 & .19| & .78 & $-.95 \mid$ & .79 & $-.90 \mid$ & |z . .32 & $.50 \mid$ & 35.7 & $34.9 \mid$ & 03522 \\
\hline 61 & 110 & 28 & .97 & $.21 \mid$ & .76 & $-.91 \mid$ & \begin{tabular}{|l|l}
.79
\end{tabular} & $-.73 \mid$ & |y .52 & .48| & 35.7 & $36.3 \mid$ & 66112 \\
\hline 104 & 109 & 28 & .93 & $.21 \mid$ & .75 & $-1.00 \mid$ & .79 & $-.77 \mid$ & |x. .55 & $.48 \mid$ & 35.7 & $36.4 \mid$ & 10312 \\
\hline 5 & 92 & 28 & .28 & .19| & .78 & $-.95 \mid$ & .77 & $-99 \mid$ & |w .58 & .58 & 35.7 & 34.9 & 6อ521 \\
\hline 56 & 121 & 28 & 1.52 & .24 & .78 & $-.67 \mid$ & .74 & $-.78 \mid$ & |v .56 & $.43 \mid$ & 35.7 & $48.1 \mid$ & 05022 \\
\hline 101 & 114 & 28 & 1.15 & .22 & .71 & -1.08 & .77 & $-.78 \mid$ & |u . .41 & $.47 \mid$ & 42.9 & 37.9 & 10112 \\
\hline 96 & 113 & 28 & 1.10 & .22 & .76 & -.89 & .76 & -.82 & |t . .70 & $.47 \mid$ & 32.1 & 38.0 & 69622 \\
\hline 53 & 124 & 28 & 1.72 & .26 & .74 & -.77 & .69 & $-.88 \mid$ & |s .61 & .41| & 53.6 & 58.0 이 & 65322 \\
\hline 72 & 117 & 28 & 1.38 & $.23 \mid$ & . 69 & -1.14 & .73 & $-91 \mid$ & $\mid r .57$ & $.45 \mid$ & 28.6 & $39.7 \mid$ & 07222 \\
\hline 18 & 99 & 28 & .53 & .19| & .69 & -1.40 & .66 & -1.54 & |q .63 & .50 & 46.4 & $35.3 \mid$ & 61821 \\
\hline 54 & 126 & 28 & 1.86 & .28 & .67 & -1.00 & .50 & -1.55 & p. .88 & .39 & 78.6 & $60.3 \mid$ & 65422 \\
\hline 34 & 111 & 28 & 1.01 & $.21 \mid$ & .58 & -1.79 & .64 & -1.41 & 0.49 & $.48 \mid$ & 57.1 & 36.21 & 03422 \\
\hline 63 & 107 & 28 & .84 & .28 & .63 & -1.62 & .56 & -1.92 & ก. .31 & .49 & 60.7 & 36.5 & 66312 \\
\hline 94 & 113 & 28 & 1.10 & $.22 \mid$ & .60 & $-1.64 \mid$ & .62 & -1.49 & |n .69 & $.47 \mid$ & 39.3 & 38.0 이 & 09412 \\
\hline 51 & 122 & 28 & 1.58 & $.25 \mid$ & .56 & -1.59 & .61 & $-1.25 \mid$ & 11.34 & $.42 \mid$ & 67.9 & $50.9 \mid$ & 05112 \\
\hline 95 & 123 & 28 & 1.65 & .26 & .59 & $-1.43 \mid$ & .52 & $-1.57 \mid$ & k. .55 & $.42 \mid$ & 67.9 & $54.8 \mid$ & 69512 \\
\hline 45 & 117 & 28 & 1.30 & $.23 \mid$ & .58 & -1.65 & .55 & -1.69 & j .16 & .45 & 64.3 & $39.7 \mid$ & 64522 \\
\hline 97 & 109 & 28 & .93 & $.21 \mid$ & .56 & -1.97 & .56 & $-1.87 \mid$ & 11.60 & .48 & 50.6 & $36.4 \mid$ & 69722 \\
\hline 36 & 95 & 28 & .39 & .19| & .55 & -2.25 & .53 & $-2.35 \mid$ & h . .42 & .50 & 42.9 & $35.1 \mid$ & 03612 \\
\hline 79 & 102 & 28 & .65 & .19| & .54 & -2.18 & .55 & $-2.69 \mid$ & lg . .59 & .50 & 46.4 & $35.4 \mid$ & 67922 \\
\hline 32 & 101 & 28 & .61 & .19| & .52 & $-2.33 \mid$ & .51 & $-2.35 \mid$ & If . .30 & .50 & 50.6 & $35.3 \mid$ & 03212 \\
\hline 81 & 122 & 28 & 1.58 & $.25 \mid$ & .43 & -2.26 & .48 & -1.82 & e .66 & $.42 \mid$ & 53.6 & $50.9 \mid$ & 98112 \\
\hline 3 & 102 & 28 & .65 & .19| & .38 & $-3.32 \mid$ & .38 & $-3.22 \mid$ & d . .68 & .58 & 60.7 & $35.4 \mid$ & 60311 \\
\hline 16 & 103 & 28 & .68 & .201 & .31 & $-3.83 \mid$ & .31 & $-3.89 \mid$ & |c .44 & .49 & 71.4 & $35.4 \mid$ & 01611 \\
\hline 17 & 102 & 28 & .65 & .19| & .26 & $-4.42 \mid$ & .26 & $-4.34 \mid$ & |b. .55 & .58 & 75.6 & $35.4 \mid$ & 01711 \\
\hline 48 & 105 & 28 & .76 & $.28 \mid$ & .26 & -4.95 & .19 & $-4.95 \mid$ & |a .76 & .49| & 78.6 & 36.6 & 64822 \\
\hline MEAN & 112.9 & 28.8 & 1.22 & $.25 \mid 1$ & 1.64 & $.0 \mid$ & 1.01 & $-.1 \mid$ & & & 47.6 & $44.3 \mid$ & \\
\hline P.SD & 12.2 &.$\theta$ & .74 & .16| & .43 & $1.5 \mid$ & .43 & $1.5 \mid$ & & & 16.8 & $11.9 \mid$ & \\
\hline
\end{tabular}


Table 8 shows the ability of students with the difficulty of each item at grain level. The criteria for checking the suitability of a person (person fit) or inconsistency of a person (outlier or misfit) are as follows: (1) an OUTFIT MNSQ value greater than 0.5 but smaller than 1.5 and closer to 1 is a good value; (2) an OUTFIT ZSTD value between -2.0 and +2.0 and closer to 0 is a good value; and (3) a value between 0.4 and 0.85 for PT MEASURE CORR is a good value. A participant can be considered fit if it meets at least 1 of these 3 criteria. Table 9 shows the instrument used for character measurement of elementary school students.

Table 9. Summary Statistics

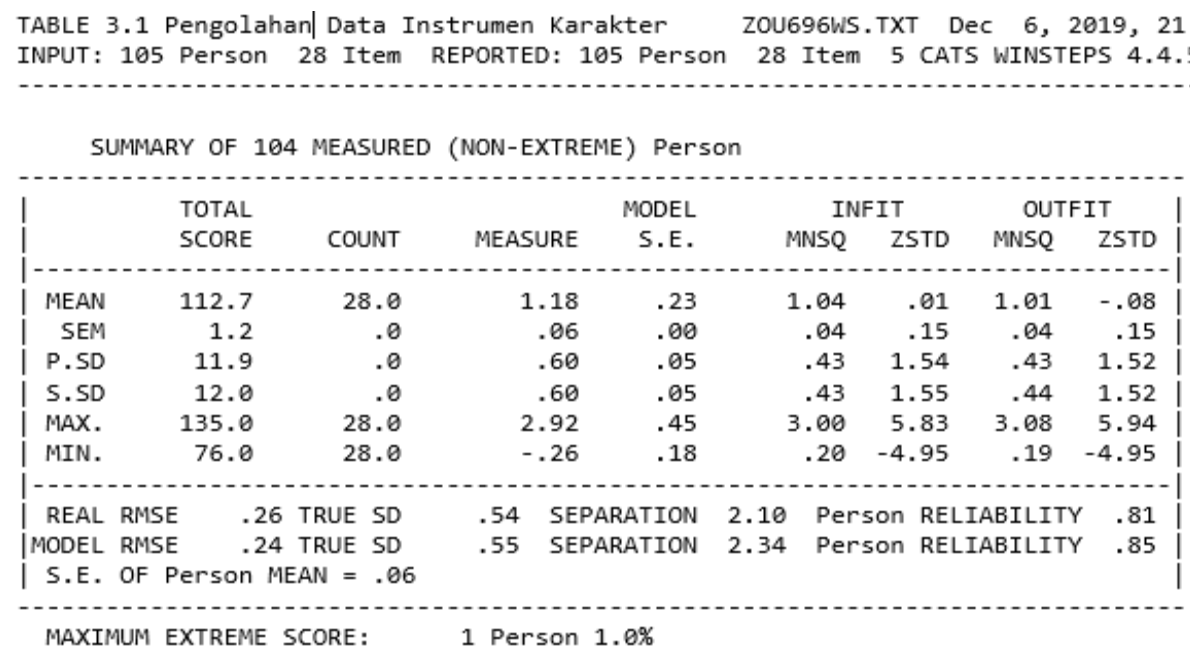

SUMMARY OF 105 MEASURED (EXTREME AND NON-EXTREME) Person

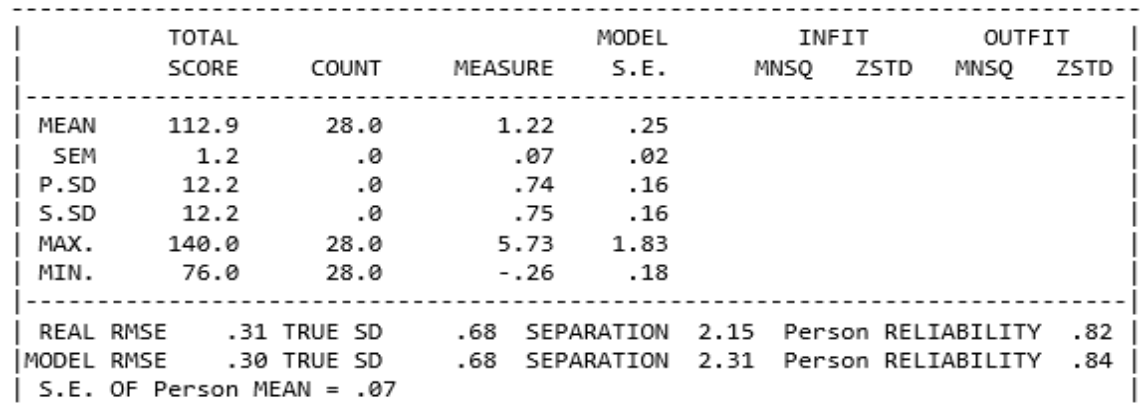

Person RAW SCORE-TO-MEASURE CORRELATION $=.90$

CRONBACH ALPHA (KR-20) Person RAW SCORE "TEST" RELIABILITY $=.85$ SEM $=4.64$

SUMMARY OF 28 MEASURED (NON-EXTREME) Item

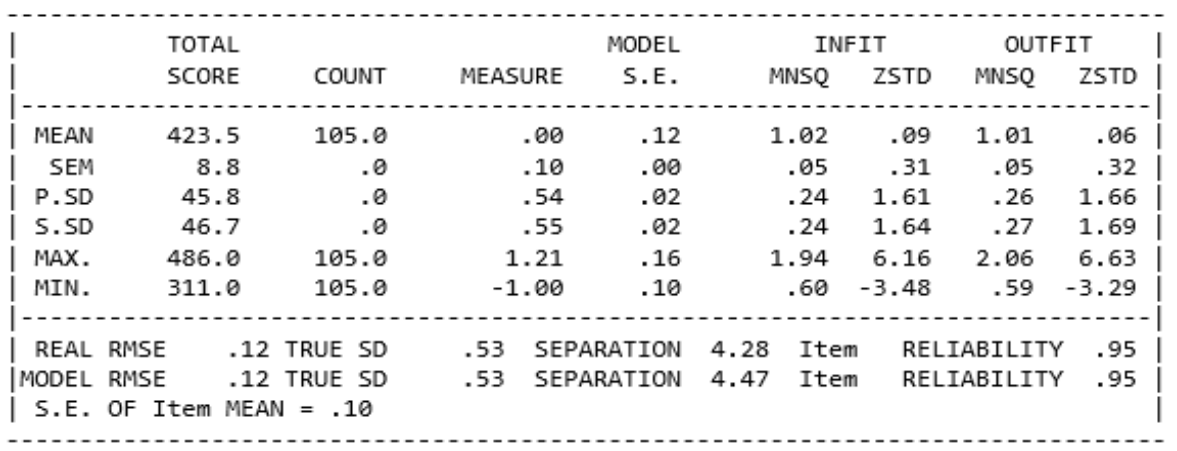

Item RAW SCORE-TO-MEASURE CORRELATION $=-.99$

Global statistics: please see Table 44 .

UMEAN $=.0000$ USCALE $=1.0000$ 
Table 10. Summary Statistics

\begin{tabular}{lccccc}
\cline { 2 - 5 } & Mean & SD & Separation & Reliability & $\begin{array}{c}\text { Cronbach } \\
\text { Alpha }\end{array}$ \\
\hline Person & 1.18 & 0.60 & 2.10 & 0.81 & \multirow{2}{*}{0.85} \\
\hline Item & 0.00 & 0.54 & 4.28 & 0.95 & \\
\hline
\end{tabular}

Referring to Table 10, reliability values for person and item are 0.81 and 0.95 . This means that the instrument (character) of the elementary school students are considered reliable within a special category. As for the Cronbach Alpha value of 0.85 , which represents the interaction between persons and items, this falls in the excellent category. This categorisation is based on Sumintono \& Widhiarso (2014) where they considered a value which is less than 0.6 to be in a bad category, a value in the range $0.6-0.7$ to be in the moderate category, a value between 0.7 and 0.8 to be in a good category and a value greater than 0.8 to be in the excellent category. There are several aspects of character in students that are different from the two schools studied. This difference occurs in one of them due to different environmental influences. SDN 1 Cikalang is located in an urban area while SDN Karsanagara is located next to an urban area. This difference can be seen in Table 6 which shows that there are some biased items, involving students at SDN 1 Cikalang and SDN Karsanagara.

\section{Discussion}

\section{Character Education in Elementary Schools}

Character can be defined as a set of individual psychological characteristics that affect one's ability and tendency to function morally (Osman, 2019) where moral is defined as the reality of personality. A positive character is a driver which will determine the right direction and acts as a protector against the occurrence of immoral actions (Fauziyah \& Jailani, 2014). Moral is not the result of personal development by itself, but includes a person's actions and behavior, as well. It is indispensable to give examples when teaching moral education. However elementary school-age students are still in the imitation stage, so it can be said indirectly that elementary school students still need something tangible so they can learn. The virtuous behavior or virtue of a person can grow through observations of other people doing such virtuous actions (Schnall et al., 2010). The discipline included in students' character can be generated through the drive to control behavior so that it can also affect the general academic performance of students (Stanley, 2014).

The teacher and his caring attitude can help students to develop positive attitudes in learning (Rahimi \& Karkami, 2015). Character development should start from the moral improvement of the teacher. Elementary school-age students are still in the imitation stage, so they still need concrete examples to copy and to learn from. Children aged 6-12 are at a concrete operational level where they still need something tangible to help develop their intellectual abilities (Desstya, 2015; Ibda, 2015). Giving concrete examples have more impact because it includes aspects of moral, cognitive, and motivational education (Mannan, 2017; Szutta, 2019). Character values raised by educators can be indirectly admired by their students. 
Zagzebski (2015) noted that someone's admiration is an emotion where the object is seen as something which is acceptable. This means that the character values shown by educators can be imitated by students through the admiration to the teacher. Character education is a process of applying moral and religious values to learners through the sciences and then applying these values to oneself, to our family, friends, educators and the surrounding environment and to God the Almighty (Putri, 2018). Character development inculcates a lot of values inside students such as being careful, thorough, ability to face problems, being honest, objectivity, perseverance and tolerance (Widodo \& Kadarwati, 2013).

Application of character education can be implemented through the Character Building (CB) subject aims at improving the quality of the students' personality so that they are ready to contribute to the community after graduation. Character education will enable them to apply many important values in life, including caring, honest, responsible, disciplined, and tolerant attitudes (Pane \& Patriana, 2016). Positive characters can grow through watching films in which students are involved in discussions to foster positive thinking and develop their character (Iii \& Waters, 2014). To achieve the goal of character education, it is therefore necessary to assume that educators are "knowledge brokers", effectively repackaging information and participating in teacher training (Walker et al., 2015).

\section{Character Instrument Development of Elementary School Students}

The development of the character instrument of the elementary school students was conducted with 28 fifth-graders of SDN 1 Cikalang and 77 sixth-graders of SDN Karsanagara. Aspects studied include interest (strong desire), beliefs (mental attitude), confidence, perseverance (persistence), fear, responsibility, discipline, honesty, tolerance, hard work, creativity, independence, curiosity, respect, social concern, love, peace, democratic, and patriotism. The study was conducted using Rasch model. This model is considered as the only model that view numbers as a truth so that the results of the analysis are considered to be authentic with adequate statistical results (Van Der Ven \& Ellis, 2000)

Table 2 (Undimensionality table) indicates that the raw variance value of 33.3\% belongs to the 'adequate' category. The value of Unexplained variance in the $1^{\text {st }}$ to the $5^{\text {th }}$ contrast of residuals is $8.0 \%, 6.2 \%, 4.3 \%, 4.2 \%$, and $4.1 \%$. All of these values are also less than $15 \%$. The use of the instrument is measured by the character variable. The general criteria for the interpretation of the variance is as follows: unexplained variance if $<15 \%$, adequate if $20 \%-40 \%$, good if $40 \%-60 \%$, and very good if above $60 \%$.

The findings of the study revealed that the majority character $(78.1 \%$ or 82 students out of 105) of elementary school students are in the medium category. This means that the character and ethics of many elementary school students still need to be improved and this can be done at school. It is proven by Table 3 and Table 7 where only 10 students were in the high-ability (positive character) category while the majority of the students are in the medium category. Thirteen students fell into the poor character value category. 
Although the results of the analysis in Table 7 showed that each student has a logit value that varies from negative to positive values, this is not a problem because the average item size has a standard value of 0.0 logit (Boone, Staver \& Yale, 2014). A negative value implies that the respondent or student has an ability level less than the average difficulty of the item the instrument is measuring. Positive characters that have not embedded in elementary school students can be influenced by several factors such as environmental factors. The surrounding environment is one of the greatest influences on human growth, starting from the family, school, and community environments.

Referring to Table 3, the difficulty level of 27 items is between $-2 \mathrm{SD}$ and $+2 \mathrm{SD}$, except for item for which it is above +2SD. The difficulty level of item 25 can be considered as an outlier. It means that this item is not an appropriate one to give to students to measure character while the other 26 items are appropriate to measure the character of elementary school students. Table 4 shows that there are 6 items that are categorized as 'very difficult'. These are items P5, P9, P17, P19, P22 and P25. There are three items that are categorized as 'difficult'. These are item P1, P2 and P8. In the 'easy category', there are 16 items namely P3, P4, P6, P7, P10, P12, P13, P14, P15, P16, P20, P21, P23, P24, P27 and P28. The 'very easy category' includes 3 items, which are P11, P18, and P28. Items within the difficult categories are aspects of character that are not possessed by students yet. These characters are devout (religious) and interest (strong desire). These two character traits are influenced by several factors including the ability of individuals to socialize with the environment, with people who have a high level of faith, with individuals who have the ability to adapt, and the ability of individuals to interact socially with the family, school and the community (Akbar, 2015).

Besides using the scales in the character instruments of elementary school students, this research also use Likert scales with a score ranges from 1 to 5 . The Likert scale were used in several questions to measure individual behavior by responding to 5 choices on each item of inquiry: strongly agree, agree, neutral, disagree, and strongly disagree (Budiaji, 2013). Four items are based on the aspect of perseverance (diligence, heart strength, persistence). Students of SDN 1 Cikalang was found to be weak in tolerance. Therefore, the fifth graders at SDN 1 Cikalang must be taught this value in more detail. Students at SDN Karsanagara had difficulty with managing their fear and things that could harm them. Therefore, they must be taught this aspect with more care and diligence and this can be delivered by the educator. These aspects are essential, giving rise to a biased tendency to one of the groups. The aspect of discipline was low for students at SDN Karsanagara. This could be explained by the fact that teachers are not giving the right examples, for example, by coming late to his class (Wardhani, 2018). This aspect of discipline is not so much a problem at SDN 1 Cikalang.

The Rasch model can provide great benefits, but researchers should evaluate it carefully and conscientiously (Boone, Staver \& Yale, 2014). Table 8 shows that 99 students have been able to answer most questions to a satisfactory extent. Only 6 students were unable to provide satisfactory answers according to their abilities. This can occur due to the possibility of students choosing random answers and 
therefore it is inaccurately measuring the character of these students. Based on the results obtained from the data collection, data processing and data analysis procedures regarding the development of the character of elementary school students, we conclude that the character development of the majority of elementary school students is in the medium category. This means that the character of elementary school students still needs much improvement through character education which must be integrated with the main subjects that are taught in elementary schools. The basic aspects of religion and interest must also be strongly emphasized. Moreover, five-graders tend to be weak in the perseverance (diligence, strength of heart, persistence) and tolerance aspects while the sixth graders tend to be weak in devout and discipline. Thus, necessary adjustments must be made to customize the teaching of character development to each group of students.

\section{Conclusions and Recommendations}

The results of data analysis using the Rasch model revealed that the character of the majority of elementary school students is in the medium category. This means that the character of elementary school students still needs to be improved through character education which needs to be integrated with the main subjects that are being taught in elementary schools. Reading, creativity, curiosity, perseverance (strength of heart) and devotion (religion) are some of the character traits that need to be emphasized in character development education. Character development of the fifth-grade students tends to be low in aspects such as perseverance (diligence, strength of heart, persistence) and tolerance while the sixth-graders tend to be weak in devotion and discipline. Based on the above findings, it can be concluded that the Rasch model is able to reveal the character of students through the use of dichotomous (multiple choice questions) data by utilizing statistical parametric tests. In other words, the Rasch model is a relevant and appropriate tool to discover the relationship between a person's ability and the difficulty level of question items.

Our final recommendations are as follows:

1. Both schools involved in this study, SDN 1 Cikalang and SDN Karsanagara, need to improve the strictness of their regulations so that they can get the desired character development traits from their students.

2. Teachers must foster positive attitudes and values in elementary school students. Teachers are central to the life of students and they have a strong influence on how many students will live their life afterwards.

3 . The community and the society at large have a vital role to play to imbue youngsters (and especially students) with a positive character by providing a environment that is conducive for such developments.

\section{Acknowledgments}

I wish to thank the lecturers of the Learning \& Evaluation course at the elementary schools who have allowed me to develop this article and provide the necessary guidance in a systematic way. A sincere thanks to all the students of SDN 1 Cikalang and SDN Karsanagara who have participated in this research. 


\section{References}

Akbar, N. (2015). Bimbingan Perkembangan Remaja yang Beriman dan Bertaqwa [Guidance for Adolescent Believers and Devotion]. Al-Hiwar: Jurnal Ilmu Dan Teknik Dakwah, 3(6), 33-38. http://dx.doi.org/10.18592/al-hiwar.v3i6.1203

Ardiyanti, D. (2017). Aplikasi Model Rasch pada Pengembangan Skala Efikasi Diri dalam Pengambilan Keputusan Karir Siswa [Application of the Rasch Model on the Development of Self-Efficiency Scale in Student Career Decision Making]. Jurnal Psikologi, 43(3), 248-263. https:// doi.org/10.22146/jpsi.17801

Aziz, R. (2015). Aplikasi Model Rasch dalam Pengujian Alat Ukur Kesehatan Mental di Tempat Kerja [Application of the Rasch Model in Testing the Mental Health Measurement Tool at Work]. Jurnal Psikoislamika, 12(2), 29-39.

Bond, T. G., \& Fox, C. M. (2015). Applying the Rasch Model: Fundamental Measurement in the Human Sciences ( $3^{\text {rd }}$ ed.). Abingdon, United Kingdom: Routledge.

Boone, W. J., Staver, J. R., \& Yale, M. S. (2014). Rasch Analysis in the Human Sciences. Amsterdam, Netherlands: Springer. https://doi.org/10.1007/978-94-007-6857-4

Budiaji, W. (2013). Skala Pengukuran dan Jumlah Respon Skala Likert [Measurement Scale and Number of Response Likert Scale]. Jurnal Ilmu Pertanian Dan Perikanan, 2(2), 127-133.

Desstya, A. (2015). Penguatan karakter siswa sekolah dasar melalui pembelajaran IPA [Strengthening the character of elementary school students through learning science]. Jurnal Aktualisasi Bimbingan Dan Konseling Pada Pendidikan Dasar Menuju Peserta Didik Yang Berkarakter, 69-75.

Ehiane, S. O. (2014). Discipline and Academic Performance (A Study of Selected secondary Schools in Lagos, Nigeria). International Journal of Academic Research in Progressive Education and Development, 3(1), 181-194. https://doi.org/10.6007/ijarped/v3i1/758

Fadilah, S. (2013). Pembentukan Karakter Siswa melalui Pembelajaran Matematika [Formation of Student Character through Mathematics Learning]. Jurnal Pendidikan Matematika, 6(2), 142-148.

Fauziyah, L., \& Jailani, J. (2014). Pengembangan Perangkat Pembelajaran Matematika yang Menunjang Pendidikan Karakter Siswa Kelas IV Sekolah Dasar [Developing Mathematic Teaching Kits That Support The Character Education of The Students In Class IV of Elementary Schools]. Jurnal Prima Edukasia, 2(2), 149-163. https://doi.org/10.1016/j.matlet.2003.12.021

Ibda, F. (2015). Perkembangan Kognitif: Teori Jean Piaget [Cognitive Development: Jean Piaget's Theory]. Jurnal Intelektualita, 3(1), 27-38.

Jackson, T. R., Draugalis, J. R., Slack, M. K., Zachry, W. M., \& Agostino, J. D. (2002). Validation of Authentic Performance Assessment: A Process Suited for Rasch Modeling. American Journal of Pharmaceutical Education, 66, 233-243.

Levin, K. A. (2006). Study design III: Cross-sectional studies. Evidence-Based Dentistry, 7(1), 24-25. https://doi.org/10.1038/sj.ebd.6400375

Makransky, G., Rogers, M. E., \& Creed, P. A. (2015). Analysis of the Construct Validity and Measurement Invariance of the Career Decision Self-Efficacy Scale: A Rasch Model Approach. Journal of Career Assessment, 23(4), 1-16. https:// doi.org/10.1177/1069072714553555

Mannan, A. (2017). Pembinaan Moral dalam Membentuk Karakter Remaja [Moral Coaching in Shaping Youth Character]. Jurnal Aqidah-Ta, 3(1), 59-72. https://doi.org/10.24252/aqidahta.v3i1.3408

Misbach, I., \& Sumintono, B. (2014). Pengembangan dan Validasi Instrumen "Persepsi Siswa terhadap Karakter Moral Guru" di Indonesia dengan Model Rasch [Development and Validation of the Instrument "Student Perceptions of the 
Moral Character of Teachers" in Indonesia with the Rasch Model]. In Seminar Nasional Psikometri, tema "Pengembangan Instrumen Penilaian Karakter yang Valid", At Solo, Indonesia.

Miller, C. B. (2013). Moral Character: An Empirical Theory. Oxford, United Kingdom: Oxford University Press.

Mubarok, H., Rusmana, N., Budiman, N., \& Suryana, D. (2019). The values of peace culture development through sociodrama. International Journal of Scientific and Technology Research, 8(11), 1453-1460.

Murniyetti, M., Engkizar, E., \& Anwar, F. (2016). Pola Pelaksanaan Pendidikan Karakter Terhadap Siswa Sekolah Dasar [The Pattern of Character Education Implementation for Elementary School Students]. Jurnal Pendidikan Karakter, 6(2), 156-166. https://doi.org/10.21831/jpk.v6i2.12045

Nur, L., Suherman, A., \& Subarjah, H. (2019). The Use of Global Positioning System (GPS) Polars to Determine Motion Intensity. Journal of Engineering Science and Technology, 14(4), 2132-2139.

Nurhudaya, N., Taufik, A., Yudha, E. S., \& Suryana, D. (2019). The Raven' s Advanced Progressive Matrices in Education Assessment with a Rasch Analysis. Universal Journal of Educational Research, 7(9), 1996-2002. https://doi.org/10.13189/ujer.2019.070921

Nurillah, S. A. L., Zerlinda, A. N., Solehuddin, M., \& Suryana, D. (2020). Analysis of Mindfulness in Elementary School. International Journal of Scientific \& Technology Research, 9(2), 1886-1890.

Osman, Y. (2019). The significance in using role models to influence primary school children's moral development: Pilot study. Journal of Moral Education, 48(3), 316331. https://doi.org/10.1080/03057240.2018.1556154

Pane, M. M., \& Patriana, R. (2016). The Significance of Environmental Contents in Character Education for Quality of Life. Procedia - Social and Behavioral Sciences, 222, 244-252. https://doi.org/10.1016/j.sbspro.2016.05.153

Purba, S. E. D. (2018). Analisis model Rasch instrumen tes prestasi pada mata pelajaran dasar dan pengukuran listrik [Rasch model analysis of achievement test instruments on basic subjects and electrical measurements]. Wiyata Dharma: Jurnal Penelitian Dan Evaluasi Pendidikan, 6(2), 142-147. https://doi.org/10.30738/wd.v6i2.3393

Putri, D. P. (2018). Pendidikan Karakter Pada Anak Sekolah Dasar di Era Digital [Character Education in Primary School Children in the Digital Age]. Ar-Riayah: Jurnal Pendidikan Dasar, 2(1), 37-50. https://doi.org/10.29240/jpd.v2i1.439

Rahimi, M., \& Karkami, F. H. (2015). The role of teachers' classroom discipline in their teaching effectiveness and students' language learning motivation and achievement: A path method. Iranian Journal of Language Teaching Research, 3(1), $57-82$.

Russell III, W. B., \& Waters, S. (2014). Developing Character in Middle School Students: A Cinematic Approach. The Clearing House: A Journal of Educational Strategies, Issues and Ideas, 87(4), 161-167. https://doi.org/10.1080/00098655.2014.888046

Salzberger, T., \& Sinkovics, R. R. (2006). Reconsidering the problem of data equivalence in international marketing research: Contrasting approaches based on CFA and the Rasch model for measurement. International Marketing Review, 23(4), 390-417. https://doi.org/10.1108/02651330610678976

Saomah, A., Suryana, D., \& Adzani, O. A. (2020). Aggressive Behavior In Elementary School. International Journal of Scientific \& Technology Research, 9(2), 1891-1894.

Schnall, S., Roper, J., \& Fessler, D. M. T. (2010). Elevation leads to altruistic behavior. Psychological Science, 21(3), 315-320. https://doi.org/10.1177/0956797609359882 
Shih, C., Wu, C., Lai, F., \& Liao, C. (2015). The Study of Teachers' Attitudes of Positive Disciplines and Class Management Effectiveness in Junior High. International Journal of Information and Education Technology, 5(7), 507-511. https:// doi.org/10.7763/IJIET.2015.V5.558

Sumintono, B., Said, H., \& Mislan, N. (2012). Constraints and Improvement: A case Study of the Indonesia's International Standard School in Improving its Capacity Building. Journal of Education and Learning, 6(1), 22-31. https://doi.org/10.11591/edulearn.v6i1.187

Sumintono, B., \& Widhiarso, W. (2014). Aplikasi Model Rasch untuk Penelitian Ilmu-Ilmu Sosial [Application of the Rasch Model for Social Sciences Research]. Bandung, Indonesia: Trimkom Publishing House.

Supraptiningrum, S., \& Agustini, A. (2015). Membangun Karakter Siswa Melalui Budaya Sekolah Di Sekolah Dasar [Building Student Character Through School Culture In Primary Schools]. Jurnal Pendidikan Karakter, 2015(2), 219-228.

Szutta, N. (2019). Exemplarist moral theory - some pros and cons. Journal of Moral Education, 48(3), 280-290. https://doi.org/10.1080/03057240.2019.1589435

Timofte, R. S., \& Siminiciuc, L. (2018). Utilisation of Rasch Model for The Analysis of an Instrument Developed by Mapping Items to Cognitive Levels of Marzano Taxonomy. Acta Didactica Napocensia, 11(2), 71-78. https://doi.org/10.24193/adn.11.2.6.72

Van Der Ven, A. H. G. S., \& Ellis, J. L. (2000). A Rasch analysis of Raven's standard progressive matrices. Personality and Individual Differences, 29(1), 45-64. https:// doi.org/10.1016/S0191-8869(99)00177-4

Wahyuni, A., Aji, A., Tias, W., \& Sani, B. (2013). Peran Etnomatematika dalam Membangun Karakter Bangsa [The Role of Ethnomatematics in Building the Nation's Character]. In Seminar Nasional Matematika dan Pendidikan Matematika dengan tema "Penguatan Peran Matematika dan Pendidikan Matematika untuk Indonesia yang Lebih Baik" (pp. 111-118).

Walker, D. I., Roberts, M. P., \& Kristjánsson, K. (2015). Towards a new era of character education in theory and in practice. Educational Review, 67(1), 79-96. https://doi.org/10.1080/00131911.2013.827631

Wardhani, M. W. (2018). Faktor-Faktor Penyebab Rendahnya Kedisiplinan Siswa SDN Kepek Pengasih Kulon Progo Yogyakarta [Factors Causing The Low Discipline of Students of Kepek Pengasih Kulon Elementary School Progo Yogyakarta]. Jurnal Pendidikan Guru Sekolah Dasar Edisi, 19(7), 1877-1886.

Widodo, T., \& Kadarwati, S. (2013). Problem-solving-based higher order thinking to improve learning achievement through students' character building orientation. Jurnal Cakrawala Pendidikan, 32(1), 161-171.

Yudha, E. S., Suryana, D., \& Nabella, Y. (2020). Dimension of Self-Esteem Based on Guidance And Counseling Perspective. International Journal of Scientific $\mathcal{E}$ Technology Research, 9(2), 1910-1913.

Zagzebski, L. (2015). Admiration and the Admirable. Aristotelian Society Supplementary Volume, 89(1), 205-221. https://doi.org/10.1111/j.1467-8349.2015.00250.x 
Character Instrument

\begin{tabular}{|c|c|c|c|c|}
\hline No & $\begin{array}{l}\text { Dimensions } \\
\text { /Aspects }\end{array}$ & Indicator & Statement & $\begin{array}{l}\text { Item } \\
\text { No. }\end{array}$ \\
\hline \multicolumn{5}{|c|}{$\begin{array}{l}\text { The development of this instrument begins from the theory that character deals with } \\
\text { distinguishing features (differentiation) which are unique (typical or single) and are } \\
\text { owned by each nation and nations' superiority to be considered and respected equally. }\end{array}$} \\
\hline \multirow[t]{2}{*}{1} & $\begin{array}{l}\text { Interest (strong } \\
\text { desire) }\end{array}$ & $\begin{array}{l}\text { A strong will or desire to a } \\
\text { particular object. }\end{array}$ & $\begin{array}{l}\text { I am interested in } \\
\text { fraction addition } \\
\text { materials. }\end{array}$ & 1 \\
\hline & & & $\begin{array}{l}\text { I want to know the } \\
\text { actual distance by using } \\
\text { the scale on the map. }\end{array}$ & 2 \\
\hline 2 & $\begin{array}{l}\text { Beliefs (mental } \\
\text { attitude associated } \\
\text { with true) }\end{array}$ & $\begin{array}{l}\text { Behavioral conception that } \\
\text { arises from the soul as a } \\
\text { reaction on the basis of the } \\
\text { situation that affects it. }\end{array}$ & $\begin{array}{l}\text { I am sure with my own } \\
\text { answer. }\end{array}$ & 3 \\
\hline 3 & $\begin{array}{l}\text { Confidence (mental } \\
\text { attitude of trust)) }\end{array}$ & $\begin{array}{l}\text { Attitude grows on the } \\
\text { basis of confidence. }\end{array}$ & $\begin{array}{l}\text { I answer the questions } \\
\text { with my own ability. }\end{array}$ & 4 \\
\hline \multirow[t]{2}{*}{4} & $\begin{array}{l}\text { Perseverance } \\
\text { (diligence, strength } \\
\text { of heart, persistence) }\end{array}$ & Behavior shows diligence. & $\begin{array}{l}\text { I work on my } \\
\text { assignments a week } \\
\text { before the due date. }\end{array}$ & 5 \\
\hline & & & $\begin{array}{l}\text { I study before getting the } \\
\text { test. }\end{array}$ & 6 \\
\hline \multicolumn{5}{|c|}{ Character is closely related to the characteristics of a nation. } \\
\hline \multirow[t]{3}{*}{1} & Devout (religious) & $\begin{array}{l}\text { Abstain from deviant } \\
\text { deeds and obey the rules } \\
\text { of his religion. }\end{array}$ & $\begin{array}{l}\text { I pray before the class } \\
\text { begins. }\end{array}$ & 7 \\
\hline & & & I pray on time. & 8 \\
\hline & & & I pray Dhuha. & 9 \\
\hline 2 & Responsible & $\begin{array}{l}\text { Self-awareness towards all } \\
\text { intentional or } \\
\text { unintentional behavior } \\
\text { and actions. }\end{array}$ & $\begin{array}{l}\text { I apologize when I make } \\
\text { mistake. }\end{array}$ & 10 \\
\hline \multirow[t]{2}{*}{3} & Disciplined & $\begin{array}{l}\text { Feelings of obedience and } \\
\text { submissive to the values } \\
\text { implemented in their } \\
\text { environment. }\end{array}$ & $\begin{array}{l}\text { I do my duty to clean the } \\
\text { classroom. }\end{array}$ & 11 \\
\hline & & & $\begin{array}{l}\text { I come to classroom on } \\
\text { time. }\end{array}$ & 12 \\
\hline \multirow[t]{2}{*}{4} & Honest & No cheating, no lying. & $\begin{array}{l}\text { I agree if any items lying } \\
\text { around will be returned } \\
\text { to their owners. }\end{array}$ & 13 \\
\hline & & & $\begin{array}{l}\text { I do my assignments } \\
\text { honestly. }\end{array}$ & 14 \\
\hline
\end{tabular}




\begin{tabular}{|c|c|c|c|c|}
\hline 5 & Tolerant & $\begin{array}{l}\text { Respect and appreciate } \\
\text { each group or each } \\
\text { individual. }\end{array}$ & $\begin{array}{l}\text { I agree to befriend with } \\
\text { someone from different } \\
\text { religion. }\end{array}$ & 15 \\
\hline 6 & Hard working & $\begin{array}{l}\text { Never be tired and never } \\
\text { stop to pursue goals. }\end{array}$ & $\begin{array}{l}\text { I try to answer the } \\
\text { questions about fraction } \\
\text { subtraction. }\end{array}$ & 16 \\
\hline 7 & Creative & $\begin{array}{l}\text { Able to bring up new } \\
\text { ideas. }\end{array}$ & $\begin{array}{l}\text { I find another way to } \\
\text { solve the question about } \\
\text { ratio. }\end{array}$ & 17 \\
\hline 8 & Independent & $\begin{array}{l}\text { Attitude of not depending } \\
\text { on others. }\end{array}$ & $\begin{array}{l}\text { I agree to come to school } \\
\text { without bothering my } \\
\text { parents. }\end{array}$ & 18 \\
\hline 9 & Curious & $\begin{array}{l}\text { Behavior of finding out } \\
\text { something, exploring, } \\
\text { investigating, and } \\
\text { learning. }\end{array}$ & $\begin{array}{l}\text { I study at home first } \\
\text { before the teacher } \\
\text { explains the fraction } \\
\text { material. }\end{array}$ & 19 \\
\hline \multirow[t]{2}{*}{10} & Respect & Appreciate & $\begin{array}{l}\text { I value the work of } \\
\text { others honestly. }\end{array}$ & 20 \\
\hline & & & $\begin{array}{l}\text { I shake hands when I } \\
\text { meet my teacher. }\end{array}$ & 21 \\
\hline 11 & Social care & $\begin{array}{l}\text { Interest or willingness in } \\
\text { helping others. }\end{array}$ & $\begin{array}{l}\text { I help my friends to } \\
\text { understand the material } \\
\text { about the fraction } \\
\text { division. }\end{array}$ & 22 \\
\hline 12 & Love peace & $\begin{array}{l}\text { Attitudes, utterances, } \\
\text { actions that make other } \\
\text { people feel safe and } \\
\text { happy. }\end{array}$ & $\begin{array}{l}\text { I agree if the teacher } \\
\text { gives rewards to } \\
\text { students who have never } \\
\text { make trouble in the class. }\end{array}$ & 23 \\
\hline 13 & Democratic & $\begin{array}{l}\text { Discuss to appoint the } \\
\text { class leader. }\end{array}$ & $\begin{array}{l}\text { I am involved in } \\
\text { determining the class } \\
\text { chairman. }\end{array}$ & 24 \\
\hline 14 & Love reading & $\begin{array}{l}\text { Reading textbooks, } \\
\text { reading novels. }\end{array}$ & $\begin{array}{l}\text { I read at least two story } \\
\text { books every week. }\end{array}$ & 25 \\
\hline \multirow[t]{3}{*}{15} & Nationalism & $\begin{array}{l}\text { Proud as an Indonesian } \\
\text { citizen, exalt nation face. } \\
\text { Proud to use Indonesian } \\
\text { products. }\end{array}$ & $\begin{array}{l}\text { I am proud to wear a red } \\
\text { and white T-shirt. }\end{array}$ & 26 \\
\hline & & & $\begin{array}{l}\text { I want to win the } \\
\text { International Math } \\
\text { Olympiad. }\end{array}$ & 27 \\
\hline & & & $\begin{array}{l}\text { I love local products } \\
\text { rather than foreign } \\
\text { products. }\end{array}$ & 28 \\
\hline
\end{tabular}



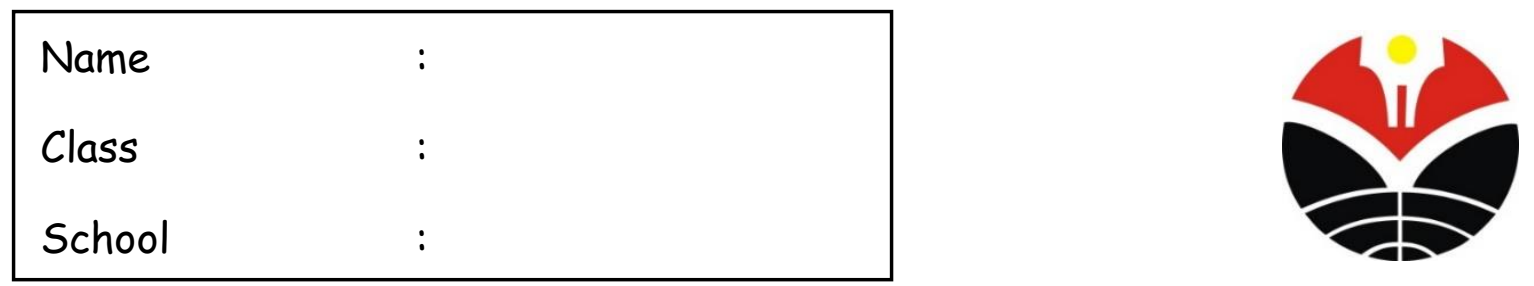

Direction:

1. Write down your identity in the available column!

2. Pray before working on the test!

3. Answer all the questions individually!

4. Cross $(X)$ one of the options!

\section{Good Luck^^}

1. I am interested in fraction addition material.
a. Not very interested
b. Not interested
c. Neutral
d. Interested
e. very interested

2. I want to know the actual distance by using the scale on the map.
a. very unwilling
b. unwilling
c. Neutral
d. willing
e. very willing

3. I am sure with my own answer.
a. very unsure
b. unsure
c. Neutral
d. Sure
e. very sure

4. I answer the questions with my own ability.
a. Never
b. Seldom
c. Sometimes
d. Often
e. Always

5. I work on my assignments a week before the due date.
a. Never
b. Seldom
c. Sometimes
d. Often
e. Always

6. I study before getting the test.
a. Never
b. Seldom
c. Sometimes
d. Often
e. Always

7. I pray before the class begins.
a. Never
b. Seldom
c. Sometimes
d. Often
e. Always

8. I pray on time.
a. Never
b. Seldom
c. Sometimes
d. Often
e. Always

9. I pray Dhuha.
a. Never
b. Seldom
c. Sometimes
d. Often
e. Always 
10. I apologize when I make mistake.
a. Never
b. Seldom
c. Sometimes
d. Often
e. Always

11. I do my duty to clean the classroom.
a. Never
b. Seldom
c. Sometimes
d. Often
e. Always

12. I come to classroom on time.
a. Never
b. Seldom
c. Sometimes
d. Often
e. Always

13. I agree if any items lying around will be returned to their owners.
a. Strongly disagree
b. Disagree
c. Neutral
d. Agree
e. Strongly agree

14. I do my assignments honestly.
a. Never
b. Seldom
c. Sometimes
d. Often
e. Always

15. I agree to befriend with someone from different religion.
a. Strongly disagree
b. Disagree
c. Neutral
d. Agree
e. Strongly agree

16. I try to answer the questions about fraction subtraction.
a. Never
b. Seldom
c. Sometimes
d. Often
e. Always

17. I find another way to solve the questions about ratio.
a. Never
b. Seldom
c. Sometimes
d. Often
e. Always

18. I agree to come to school without bothering my parents.
a. Strongly disagree
b. Disagree
c. Neutral
d. Agree
e. Strongly agree

19. I study at home first before the teacher explains the fraction material.
a. Never
b. Seldom
c. Sometimes
d. Often
e. Always

20. I value the work of others honestly.
a. Never
b. Seldom
c. Sometimes
d. Often
e. Always

21. I shake hands when I meet my teacher.
a. Never
b. Seldom
c. Sometimes
d. Often
e. Always 
22. I help my friends to understand the material about the fraction division.
a. Never
b. Seldom
c. Sometimes
d. Often
e. Always

23. I agree if the teacher give rewards to the students who never make trouble in the class.
a. Strongly disagree
b. Disagree
c. Neutral
d. Agree
e. Strongly agree

24. I am involved in determining the class chairman.
a. Never
b. Seldom
c. Sometimes
d. Often
e. Always

25. I read at least two story books every week.
a. Never
b. Seldom
c. Sometimes
d. Often
e. Always

26. I am proud to wear a red and white T-shirt.
a. Strongly disagree
b. Disagree
c. Neutral
d. Agree
e. Strongly agree

27. I want to win the International Math Olympiad.
a. very unwilling
b. unwilling
c. Neutral
d. willing
e. very willing

28. I love local products rather than foreign products.
a. Strongly disagree
b. Disagree
c. Neutral
d. Agree
e. Strongly agree 